\title{
An Automated High Performance Capillary Liquid Chromatography-Fourier Transform Ion Cyclotron Resonance Mass Spectrometer for High-Throughput Proteomics
}

\author{
Mikhail E. Belov, ${ }^{*}$ Gordon A. Anderson, Mark A. Wingerd, \\ Harold R. Udseth, Keqi Tang, David C. Prior, Kenneth R. Swanson, \\ Michael A. Buschbach, Eric F. Strittmatter, Ronald J. Moore, \\ and Richard D. Smith
}

Biological Sciences Division and Environmental Molecular Sciences Laboratory, Pacific Northwest National Laboratory, Richland, Washington, USA

\begin{abstract}
We describe a fully automated high performance liquid chromatography 9.4 tesla Fourier transform ion resonance cyclotron (FTICR) mass spectrometer system designed for proteomics research. A synergistic suite of ion introduction and manipulation technologies were developed and integrated as a high-performance front-end to a commercial Bruker Daltonics FTICR instrument. The developments incorporated included a dual-ESI-emitter ion source; a dualchannel electrodynamic ion funnel; tandem quadrupoles for collisional cooling and focusing, ion selection, and ion accumulation, and served to significantly improve the sensitivity, dynamic range, and mass measurement accuracy of the mass spectrometer. In addition, a novel technique for accumulating ions in the ICR cell was developed that improved both resolution and mass measurement accuracy. A new calibration methodology is also described where calibrant ions are introduced and controlled via a separate channel of the dual-channel ion funnel, allowing calibrant species to be introduced to sample spectra on a real-time basis, if needed. We also report on overall instrument automation developments that facilitate high-throughput and unattended operation. These included an automated version of the previously reported very high resolution, high pressure reversed phase gradient capillary liquid chromatography (LC) system as the separations component. A commercial autosampler was integrated to facilitate $24 \mathrm{~h} /$ day operation. Unattended operation of the instrument revealed exceptional overall performance: Reproducibility (1-5\% deviation in uncorrected elution times), repeatability $(<20 \%$ deviation in detected abundances for more abundant peptides from the same aliquot analyzed a few weeks apart), and robustness (high-throughput operation for 5 months without significant downtime). When combined with modulated-ionenergy gated trapping, the dynamic calibration of FTICR mass spectra provided decreased mass measurement errors for peptide identifications in conjunction with high resolution capillary LC separations over a dynamic range of peptide peak intensities for each spectrum of $10^{3}$, and $>10^{5}$ for peptide abundances in the overall separation. (J Am Soc Mass Spectrom 2004, 15, 212-232) (C) 2004 American Society for Mass Spectrometry
\end{abstract}

$\mathrm{O}$ ne of the most challenging endeavors in modern biological science is to understand the operations of living cells at the molecular level. It is clear that advanced genome sequence information provides only initial and incomplete (and often misleading) predictions of protein function(s) [1] for this most functionally important subset of biomolecules,

Published online December 10, 2003

Address reprint requests to Dr. R. D. Smith, Environmental and Molecular Sciences Laboratory, Pacific Northwest National Laboratory, MSIN K8-98, P.O. Box 999, Richland, WA 99352, USA E-mail: rds@pnl.gov

*Current address: Biospect Inc., 951 Gateway Blvd., South San Francisco, CA 94080 and that new high-throughput tools for more comprehensive studies at the protein level would benefit many areas of biological research. After the initial report by O'Farrell [2], polyacrylamide two-dimensional polyacrylamide gel electrophoresis (2D PAGE) became the dominant approach for the study of complex protein mixtures. Identification of protein spots from 2D PAGE has been greatly advanced by developments in mass spectrometry, including matrix-assisted laser desorption/ionization (MALDI) $[3,4]$ and electrospray ionization (ESI) [5-7]. Modern technology features robotic cutting of protein spots from a $2 \mathrm{D}$ PAGE followed by automated enzymatic digestion and 
transfer for analysis by MALDI-MS or ESI-MS or MS/ MS.

However, the sensitivity of 2D PAGE is limited by the need to visualize a protein spot on the gel and by the necessary sample processing prior to mass spectrometric characterization. Though identification of proteins from 2D PAGE is now often feasible at femtomole levels [8-11], many important regulatory proteins are expressed at low concentrations and are often present in abundances too low for effective study. 2D PAGE is limited further by bias toward higher abundance proteins and against proteins with extremes in $\mathrm{pI}$ and MW as well as against membrane-associated or bound proteins. Gygi et al. [12] showed that the number of 2D PAGE "spots" is poorly correlated with the number of different proteins actually detected, and that those identified were expected to be present predominantly at high abundance based on their codon adaptation indices (CAI) [13].

While efforts to enhance the capabilities of 2D PAGE are underway, non-gel-based approaches for proteomic studies are being increasingly pursued [14]. Link et al. [15] reported unbiased peptide-level identification of proteins from yeast (Saccharomyces cerevisiae), including many proteins with extremes in $\mathrm{pI}$, MW, abundance, and hydrophobicity. Yates and co-workers [16] applied their multidimensional LC-MS/MS protein identification technology (MudPIT) to identify 1484 proteins from yeast. The MudPIT approach is based on loading complex peptide mixtures from different fractions of bacterial whole-cell lysate onto a biphasic microcapillary column packed with strong cation exchange and reverse-phase materials. Peptides eluting from the capillary are analyzed by ESI-MS/MS using an ion trap mass spectrometer. The majority of proteins identified from $S$. cerevisiae had CAIs $<0.2$, in accord with the predicted distribution of proteins from the yeast genome. In contrast, few proteins with CAIs $<0.2$ were detected by 2D PAGE [17]. Similarly, discrimination against proteins with extremes in $\mathrm{pI}$ and MW is effectively eliminated.

Despite these advantages over the classical 2D PAGE approach, the MudPIT approach has shortcomings. First, the LC peak capacity or proteome coverage is limited by the rate at which the mass spectrometer can switch between MS and MS/MS modes and in the number of co-eluting species that can be analyzed.

Beyond a rather low limit of sample complexity, and thus complexity of the co-eluting peptides, any increase in sample complexity must be accompanied by a longer overall separations times if the full complement of peptides are to be analyzed by MS/MS. This is necessary to avoid significant "undersampling" of eluting peptides. Correspondingly, the two-dimensional separations and the ion-trap detection scheme used in the MudPIT approach produce analysis times of $>30 \mathrm{~h}$ for a single sample of global tryptic digest. However, even with such extended analysis times, repetitive analyses reveal substantial increases in the number of peptides identified, indicating that detected peptides in the LC eluent remain significantly under-sampled by MS/MS.

A second issue relates to the confidence of peptide/ protein identifications. Tryptic peptides are typically identified from MS/MS data using programs such as SEQUEST [18]. The general approach is based upon comparing a theoretical set of peaks predicted on the basis of the known most common peptide fragmentation processes with the experimental spectrum to produce similarity "scores". Although improved methods for scoring and data analysis continue to be developed, such scores invariably span the range from low scores where identifications are highly doubtful to high scores where identifications are quite reliable, with no clear line of demarcation. If one selects only the highest scores to reduce false positives, substantially fewer proteins will be identified. Conventionally, many MS/MS spectra and search results are manually examined to evaluate both spectrum quality and the ranking of peptide scores, generally resulting in the discarding of a substantial fraction of tentatively identified peptides; the result is improved but less quantifiable levels of confidence from the ensuing identifications. Using various statistical and more automated approaches with these tools can improve confidence in identifications, but cannot completely address the issue. In this regard, the use of highly accurate mass measurements provides an additional and high quality "test" for a tentative peptide, and thus increases confidence in identifications [19].

When coupled to capillary LC separation [20], FTICR mass spectrometry [21, 22] has been shown to be an ultra-sensitive approach with high dynamic range, mass resolution, and mass accuracy for characterization of proteolytic digests [23-26]. The sensitivity provided by FTICR has been shown to be increased by ion trapping and accumulation in a 2D rf-only octopole trap positioned external to an FTICR mass spectrometer [27]. The usefulness of ion preselection with FTICR was originally recognized by McIver [28], who proposed using a quadrupole ion guide operating at an elevated pressure. This quadrupole could prove useful for quadrupole mass filtering (rf/dc) of ions generated by an external ion source. The ions could be trapped in the FTICR cell using gas-assisted accumulated trapping [29]. The development of selective external ion accumulation $[23,30]$, where the most abundant ion species can be selectively ejected using either quadrupole mass filtering or rf-only resonant dipolar excitation [31, 32] prior to ion accumulation, enhanced the achievable FTICR detection limit to $\sim 20 \mathrm{zmol}$ ( $\sim 12,000$ molecules) for protein measurements [23]. Quenzer et al. [33] reported nano-LC-FTICR detection of $100 \mathrm{amol} \mathrm{of} \mathrm{arg}^{8}$ vasotocin in water ( $1 \mu \mathrm{L}$ of sample loaded, $100 \mathrm{amol} /$ $\mu \mathrm{L})$. Belov et al. [34] recently introduced a dynamic range enhancement applied to mass spectrometry (DREAMS) technology that provides, for example, greatly enhanced characterization of peptides in the course of a capillary LC separation of proteomic digests. 


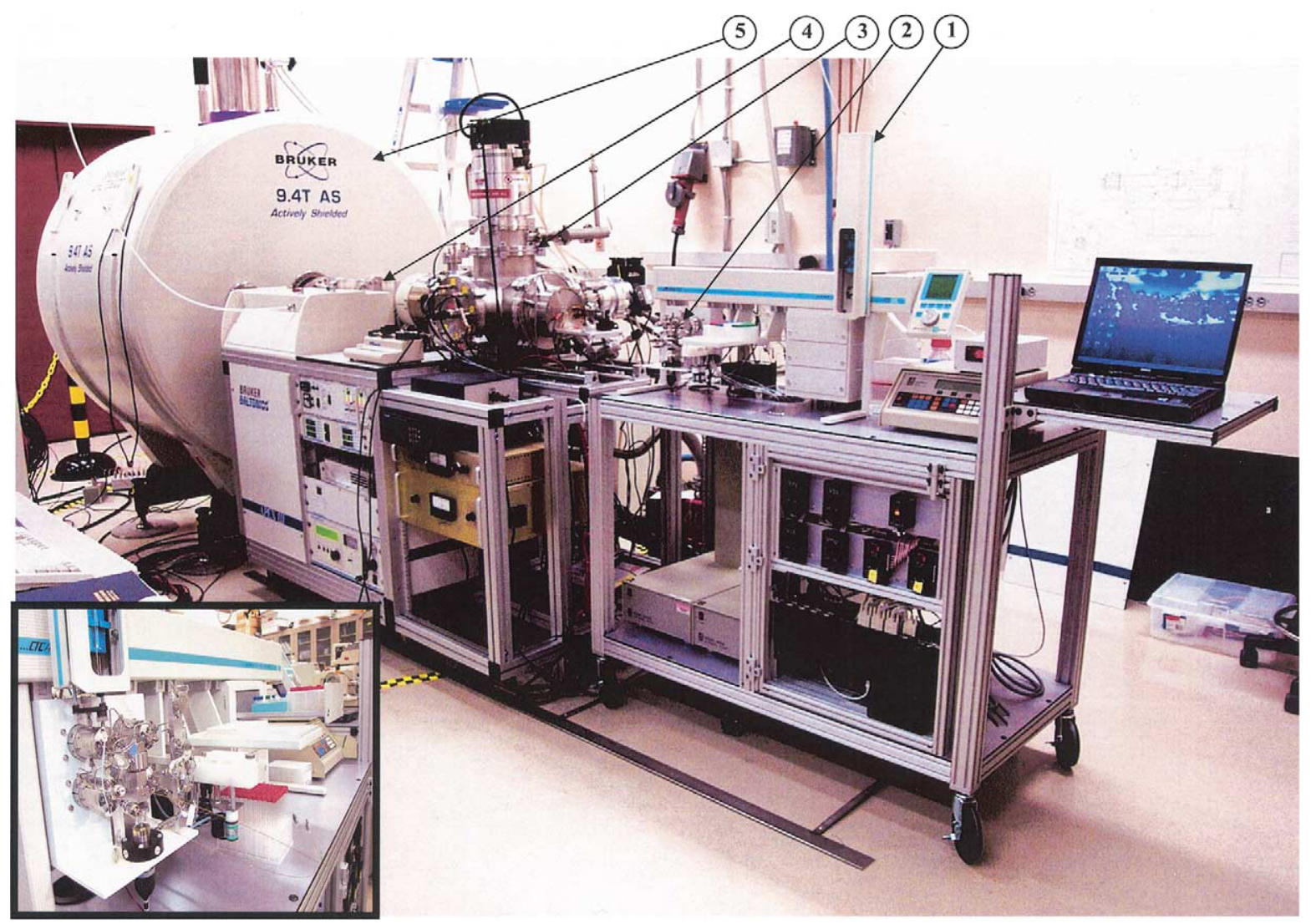

Figure 1. Photograph of the automated LC-FTICR instrument incorporating a 9.4 tesla superconducting magnet. Depicted in this photograph are: (1) The PAL autosampler, (2) the capillary LC system with injection valves, (3) the new front-end interface, (4) the commercial electrostatic ion guide, (5) the magnet. The Inset demonstrates injector valves and dual-capillary LC system.

This approach requires detection of a mass spectrum acquired during the non-selective accumulation and software-controlled selection of the $\mathrm{m} / \mathrm{z}$ of the most abundant ion peaks based on their quadrupole secular frequencies, followed by selective rf-only ejection of the most abundant species prior to external accumulation in the next scan immediately following the non-selective accumulation. Removal of these major species and the subsequent accumulation of lower abundance species for extended periods results in an increase in dynamic range. DREAMS expanded the dynamic range of FTICR to $>10^{5}$, resulting in a $40 \%$ increase over conventional LC/FTICR detection in the number of identified putative peptides from a yeast tryptic digest $[34,35]$. Shi et al. [36] showed that higher mass resolution can be achieved by lowering trapping potentials to produce a very shallow trapping well and the minimization of space charge (e.g., Coulomb repulsion) effects. FTICR has been shown to attain an extremely high mass precision of $69 \times 10^{-8} \mathrm{Da}$ in experiments with electronionized ${ }^{20} \mathrm{Ne}$ [37] and errors averaging $80 \mathrm{ppb}$ for peptides with $\mathrm{m} / \mathrm{z}$ ranging from 500 to 1150 [38], and good performance in related applications [39]. Importantly, the above mass resolution, precision, and accuracy performances were all obtained by trapping low and well-controlled ion populations in the FTICR cell.
The use of capillary LC separation in conjunction with FTICR for analysis of complex proteolytic digests (i.e., a mammalian proteome) poses a major challenge for accurate mass measurements since protein concentrations of interest in, for example, yeast vary by $>10,000$-fold [40, 41]. Therefore, LC peak intensities and the concentrations of eluted peptide mixtures span several orders of magnitude and result in large changes in ion populations in the ICR cell, causing cyclotron frequency shifts and greater mass measurement errors. To address this issue we have recently reported on data-dependent adjustment of ion accumulation times, a form of automated gain control (AGC), combined with new internal and dynamic calibration methods for ICR measurements [42]. Implementation of this approach resulted in a 5-fold reduction of mass measurement errors for higher abundance peptides identified in multiple scans during capillary LC separations compared with the conventional approach of using a fixed ion accumulation time. The use of higher magnetic field instrumentation will further decrease the dispersion of mass measurement errors for a given ion population level.

Given the aforementioned capabilities of FTICR, we developed a new approach for identifying proteins that exploited its capability for extremely high mass accuracy measurements, particularly advantageous when a 
particular biological system is to be examined many times (e.g., time course studies following perturbations) [43]. The approach initially involved conventional capillary LC-MS/MS studies to yield identified peptide "potential mass and time tags" (PMT tags) that were subsequently validated as "accurate mass and time tags" (AMT tags) if the predicted peptides' accurate masses were observed using FTICR (in a corresponding sample) at an equivalent elution time [44]. These peptide AMT tags thus serve as confident biomarkers to identify peptides/proteins in subsequent studies, and result in much greater throughput. The complexity and diversity of proteomes presents great challenges for high-throughput measurements that are aimed to be as comprehensive as possible. The use of AMT tags potentially obviates, or at least greatly reduces, the need for MS/MS and thus decreases sample size requirements. This approach is particularly attractive where multiple measurements are to be made from the same "system" (e.g., from related tissue samples). One can measure masses for tens to hundreds of peptides in each mass spectrum obtained during LC-FTICR analyses with each spectrum requiring an equal or less time than one MS/MS measurement. The resulting increase in throughput is thus, at a very minimum, equal to the average number of peptides detected in each spectrum.

In this work we describe a fully automated 9.4 tesla FTICR mass spectrometer coupled to very high pressure capillary reverse-phase liquid chromatography for high-throughput proteomic studies. The capillary LC system was designed to provide very high-efficiency separations using long packed capillaries and was implemented with an autosampler and related capabilities that enabled $24 \mathrm{~h} /$ day operation. The modifications made to the "front-end" of a commercial FTICR mass spectrometer significantly improved the sensitivity, dynamic range, and mass measurement accuracy and increased robustness and reproducibility of measurements in high-throughput analyses.

\section{Experimental}

\section{FTICR Instrumentation}

The objective of the new design was to synergistically integrate and optimize a suite of technologies developed at our laboratory for practical LC/FTICR proteomics analyses, and to do so in conjunction with very highefficiency LC separations. Further, it was desired that the design and integration of these technologies must be sufficiently robust and reliable to withstand the rigors of both high-throughput analyses and 24/7 operation.

The FTICR mass spectrometer used in this study is based on a commercial Apex III instrument (Bruker Daltonics, Billerica, MA) coupled to an actively shielded 6 in.-bore 9.4 tesla superconducting magnet. Major modifications were made to the "front-end" and interface of the commercial spectrometer which was replaced with the design shown in Figures 1 and 2. The technologies included an electrodynamic ion funnel [45], selective ion accumulation external to the ICR cell [30], DREAMS [34], and AGC with internal calibration [42]. The compactness of the commercial instrument dictated certain constraints in the design of the frontend interface of the FTICR instrument. The new interface comprising a dual-channel electrodynamic ion funnel and an assembly of rf-only quadrupoles employed for collisional focusing, ion preselection and external (to the ICR cell) accumulation of electrospray-generated ions was positioned in a standard 5-way 10 in.-o.d. vacuum cross (Nor-Cal, Yreka, CA). The vacuum region inside of the cross was evacuated by a $550 \mathrm{~L} / \mathrm{s}$ turbo pump (Varian, Lexington, MA). Since the ion funnel, collisional, and selection/accumulation quadrupoles operate in three different pressure regions, an arrangement of enclosed vacuum crosses was employed. The collisional quadrupole was placed in a standard 4-way 4 in.-o.d. vacuum cross inside of the larger 5-way 10 in.-o.d. cross, and differentially pumped by a $250 \mathrm{~L} / \mathrm{s}$ turbo pump (BOC Edwards, Crawley, Sussex, UK) to a pressure of $7 \times 10^{-3}$ torr. The ion funnel enclosure was evacuated to a pressure of $\sim 1$ torr by an $11 \mathrm{~L} / \mathrm{s}$ two-stage mechanical pump (BOC Edwards, Crawley, Sussex, UK). Both the ion selection and accumulation quadrupoles operated at a pressure of $\sim 10^{-5}$ torr. A dual ESI source was designed to independently and automatically introduce analyte and internal calibrant ions during capillary LC separations. The ESI source encompassed two ESI emitters that sprayed into two $0.4 \mathrm{~mm}$ i.d., 100 mm-long stainless steel heated inlet capillaries separated by $1 \mathrm{~cm}$. The heated capillaries used for ESI droplet desolvation and reducing the pressure from atmospheric to few torr in the first pumping region, had exits positioned flush with the first electrode of the ion funnel.

The ion funnel was modified from the design earlier reported [45] to provide independent control over the transmission of both analyte and internal calibrant ions. The ion funnel consisted of a stacked ring assembly of 100 electrodes, each $0.5 \mathrm{~mm}$ thick. The front-end of the ion funnel had 14 electrodes with identical aperture diameters of $25 \mathrm{~mm}$. An assembly of the next three electrodes comprised two independent channels for controlling ion transmission. The first and third electrodes of that assembly had two $14.3 \mathrm{~mm}$-diameter and $9.1 \mathrm{~mm}$-diameter apertures drilled $14 \mathrm{~mm}$ apart. The second electrode was split into halves, and the smaller diameter aperture was reduced to $5 \mathrm{~mm}$ to increase the contribution of a potential applied to this half plate (hereafter referred to as the "ion disrupter" electrode) to affect the potential on the aperture axis. The positioning of the ion disrupter was designed to provide ion sampling just downstream of the Mach disk formed by a supersonic jet expanding from the calibrant inlet capillary. The next 28 electrodes positioned downstream of the three-electrode-assembly had an aperture diameter of $25 \mathrm{~mm}$ followed by the converging part of the ion funnel with an angle of $30^{\circ}$ and an exit aperture of $2 \mathrm{~mm}$. Except for the ion disrupter and the exit funnel 


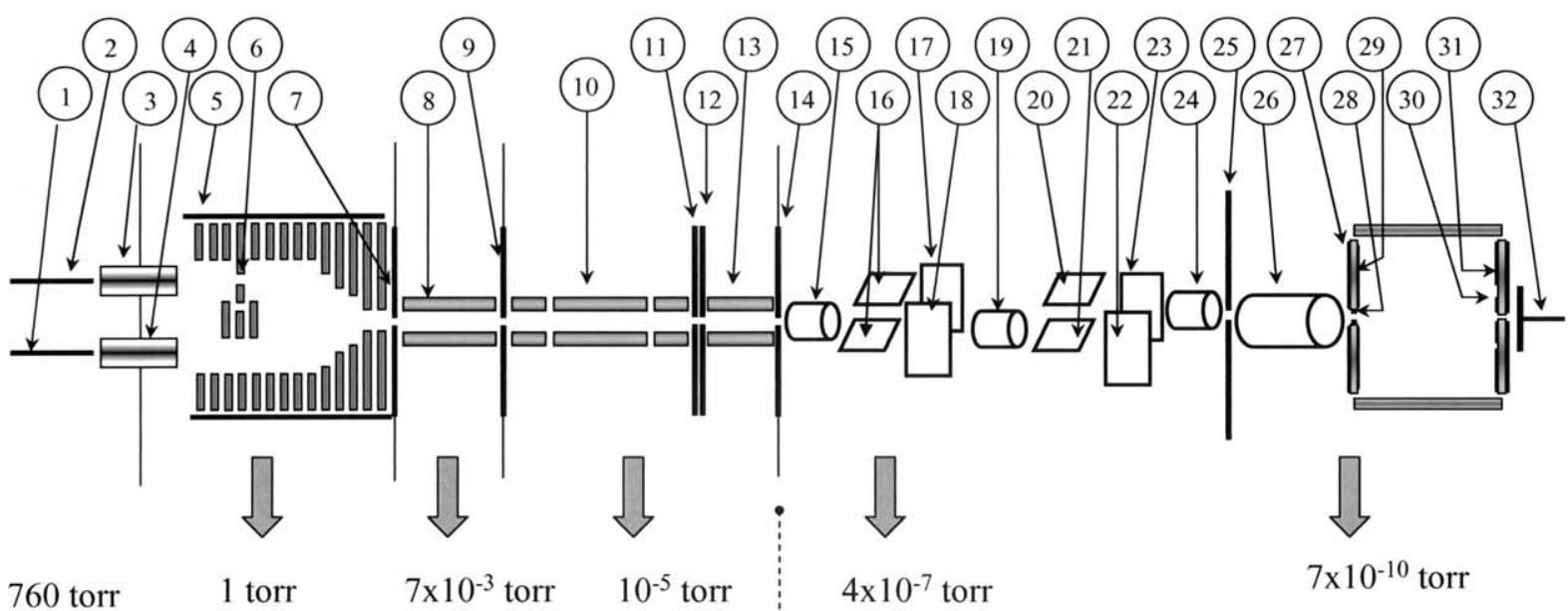

760 torr $\quad 1$ torr $\quad 7 \times 10^{-3}$ torr $10^{-5}$ torr

$\longleftarrow \quad$ New ion introduction $\longrightarrow$

(a) "front-end"

Bruker Daltonics ion Guide and ICR cell
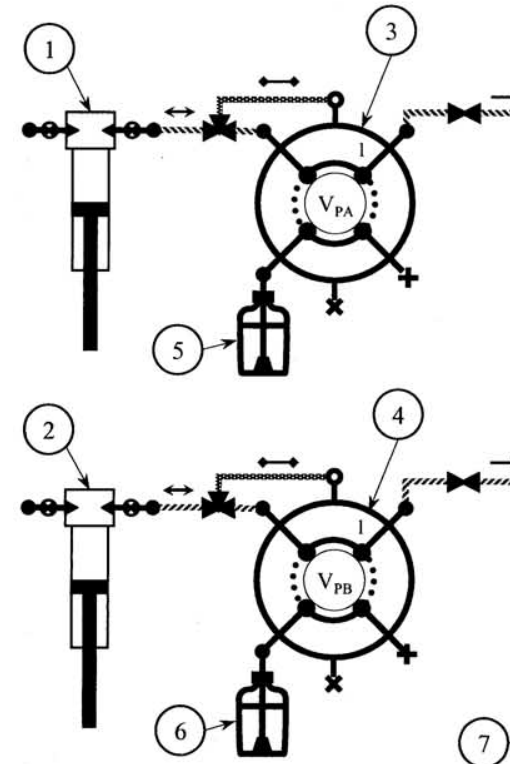

(b)

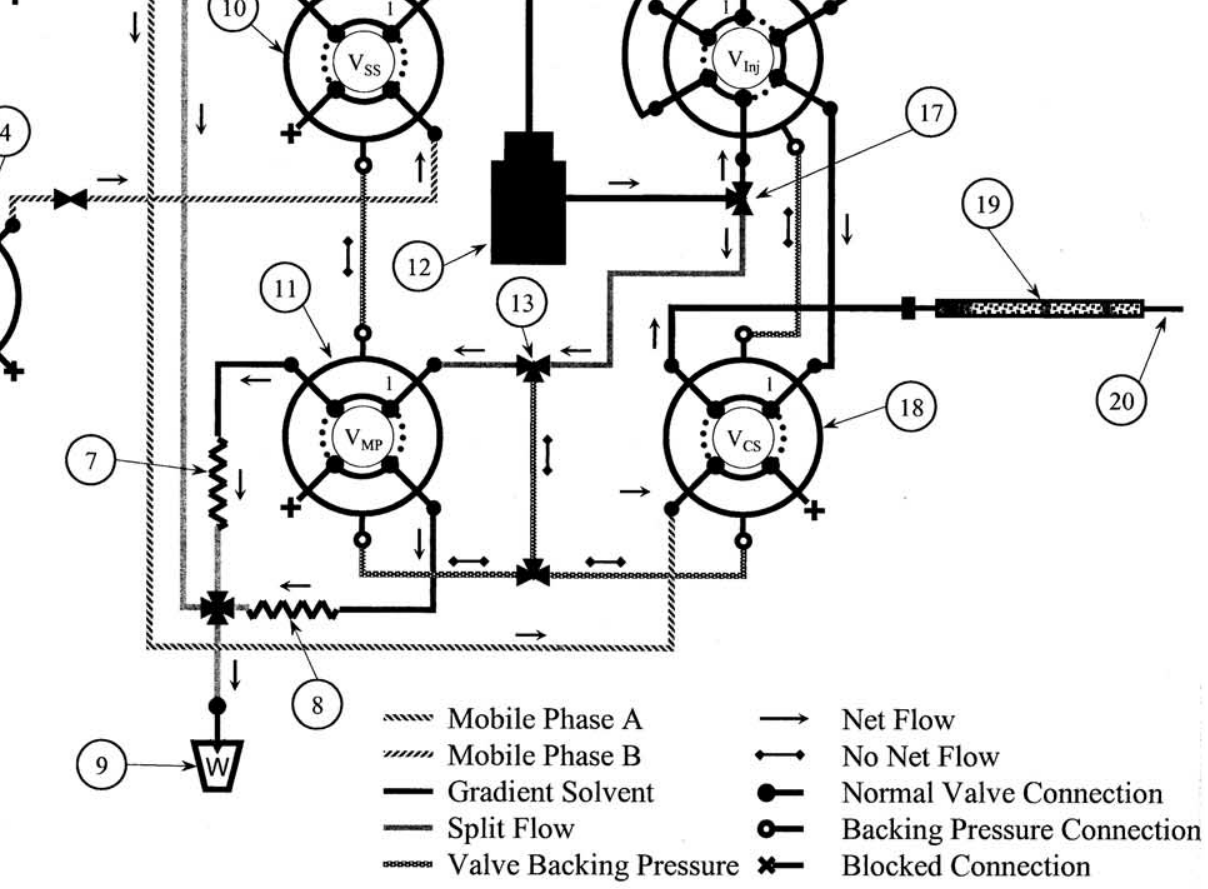

Figure 2. (a) Schematic diagram of the FTICR instrument. The elements of the ESI source: (1) The analyte ESI emitter tip, (2) the calibrant ESI emitter tip, (3) the calibrant heated inlet capillary, (4) the analyte heated inlet capillary, (5) the dual-channel ion funnel. The elements of the front-end interface that includes the electrodynamic ion funnel, collisional, selection and accumulation quadrupoles: (6) Ion disrupter electrode, (7) ion funnel aperture, (8) collisional focusing quadrupole, (9) conductance limit, (10) selection quadrupole, (11) selection quadrupole exit electrode, (12) accumulation quadru- 

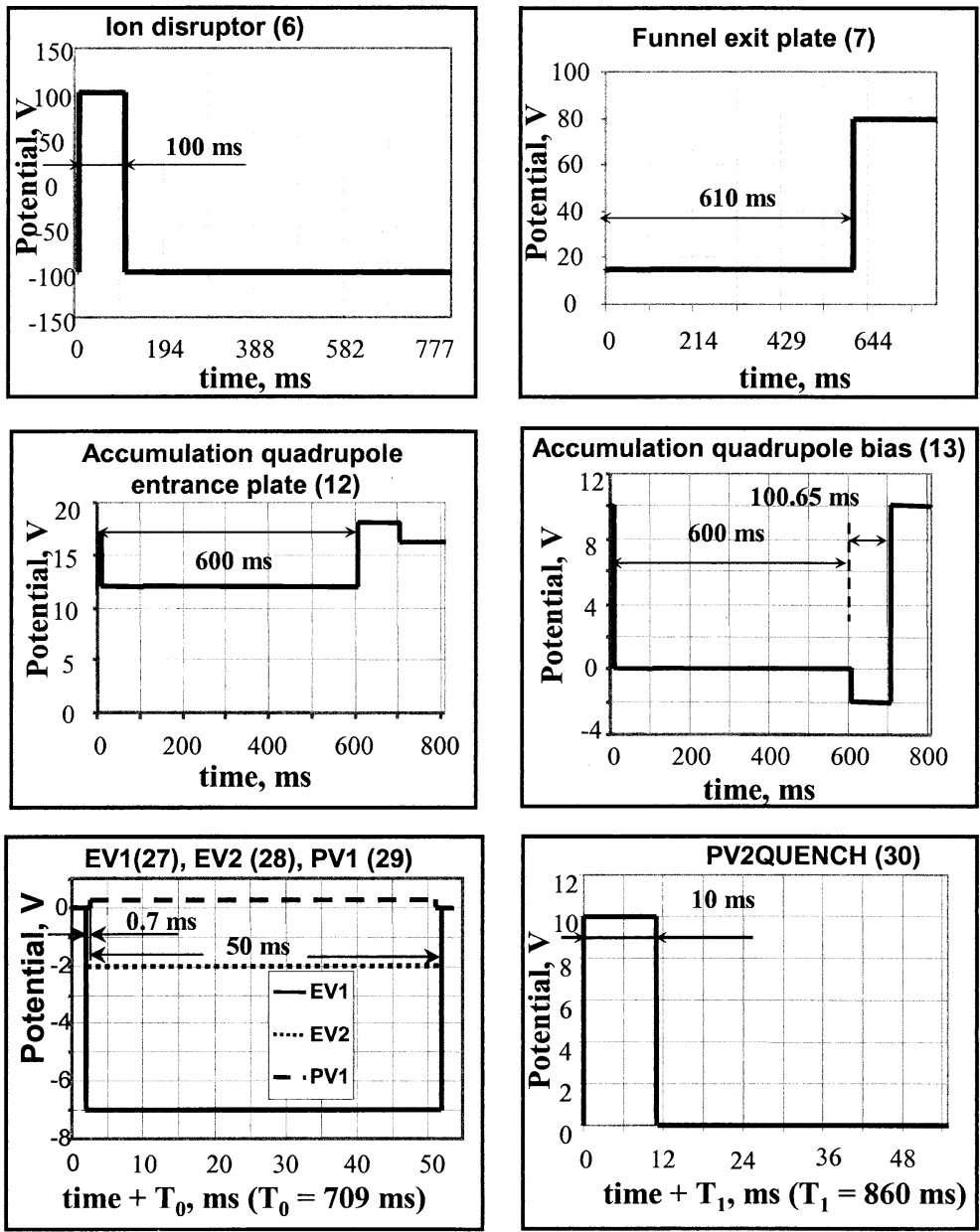

Figure 3. Timing diagrams for the pulsed voltage at different elements of the FTICR instrument. The ion optical elements are labeled in accord with Figure 2. ARB: Programmable arbitrary waveform generator; ADC: Analog-to-digital converter.

plate, a peak-to-peak rf-potential of $175 \mathrm{~V}$ was applied to all ion funnel electrodes using a built-in-house high-Q head with resonance at $360 \mathrm{kHz}$. The rf-potential polarity was alternated at the adjacent electrodes of the ion funnel to provide radial confinement of the ion beam. A dc-voltage gradient from 150 to $10 \mathrm{~V}$ was applied between the funnel entrance and exit electrodes. The potential of the ion disrupter electrode was pulsed using a built-in-house fast solid-state relay switching the dc-voltages generated by a 12-channel LeCroy dc-power supply. The timing for switching the dc-voltages was determined by the Bruker data station running the XMASS 6.0 program. Figure 3 shows the time dependences of the pulsed voltages applied to different elements of the instrument front-end interface and ICR cell through the experimental cycle.

Figure 2. (continued) pole entrance electrode, (13) ion accumulation quadrupole, (14) ion accumulation quadrupole exit electrode. Electrostatic ion guide components: (15) PL1 tube, (16) PL2 deflectors, (17 and 18) PL4 and DPL4 horizontal defector elements providing bias and deflection, respectively, (19) HVO tube, (20 and 21) XDFL vertical deflectors, (22 and 23) the YDFL horizontal deflectors, (24) FOCL1 tube, (25) PL9 lens/conductance limit, (26) the FOCL2 tube. The elements of the ICR cell: (27) EV1 element outside of the front trapping plate, (28) EV2 (bias) and DEV2 (deflection) elements at the "side-kick" electrodes, (29) PV1 element inside of the front trapping plate electrode, (30) PV2QUENCH (quench) element at the rear trapping plate, (31) PV2 element at the rear trapping plate, (32) charge collector. (b) Schematic of the high-efficiency capillary LC fluidics system: (1) Pump A, (2) Pump B, (3) Pump A control valve $\left(v_{p A}\right)$, (4) Pump B control valve $\left(v_{p B}\right)$, (5) mobile phase A reservoir, (6) mobile phase B reservoir, (7) by- pass column, LC run, (8) by-pass column, mixer purge, (9) waste line, (10) mobile phase solvent selection valve $\left(\mathrm{v}_{\mathrm{ss}}\right),(11)$ mixer purge valve $\left(\mathrm{v}_{\mathrm{mp}}\right),(12)$ mixer/dilution chamber, (13) tap point for valve backing pressure, (14) auto-sampler syringe, (15) injection port, (16) injection valve $\left(\mathrm{v}_{\text {inj }}\right)$, (17) flow split point, (18) column selection valve $\left(\mathrm{v}_{\mathrm{cs}}\right),(19)$ analytical column, (20) electrospray ionization emitter (tip). 
The ion funnel was operated in three different modes: (1) Transmission of both the analytes and calibrants, (2) transmission of only the analyte ions with efficient blocking of the calibrants, and (3) the complete blocking of transmission for both the analytes and calibrants. These regimes were accomplished by setting the ion disrupter and the exit funnel plate at (1) 120 and $10 \mathrm{~V},(2)-100$ and $10 \mathrm{~V}$, and (3) 120 and $80 \mathrm{~V}$, respectively. The funnel exit plate was pulsed in fashion similar to the accumulation quadrupole elements, as described below. Note that this ion funnel design differs from the earlier reported design with a "jet disrupter" [46]. When attempting to block the calibrant ions over an $m / z$ range of 622 Da to 2122 Da with a jet disrupter, we found that transmission efficiency through this channel of the ion funnel was somewhat $m / z$-dependent with higher $m / z$ ions being "disrupted" more readily than their lower $m / z$ counterparts. This behavior is similar to the gas dynamic focusing of heavier species in a supersonic jet [47]. A further increase in the potential applied to the jet disrupter plate resulted in decreased transmission and increased intensity variations of the analyte species. Electrical isolation between the "ion disrupter" and the half plate in the analyte channel ensured no effect on the analyte transmission at any blocking potential applied to the ion disrupter. The 150 mm-long collisional, $205 \mathrm{~mm}$-long selection, and 50 mm-long accumulation quadrupoles had an inscribed radius of $4.1 \mathrm{~mm}$ at a rod diameter of $9.52 \mathrm{~mm}$ and were driven by the same high-Q head at a frequency of 560 $\mathrm{kHz}$. A peak-to-peak rf-potential of $460 \mathrm{~V}$ was applied to the collisional and selection quadrupole rods biased to 10 and $6 \mathrm{~V}$, respectively. A conductance limit with an aperture diameter of $2 \mathrm{~mm}$ separated the collisional and selection quadrupoles. Each selection quadrupole rod was separated into three sections, $10 \mathrm{~mm}$-long end segments and a $185 \mathrm{~mm}$-long middle segment. An auxiliary rf-voltage at a peak-to-peak amplitude of $<1$ $\mathrm{V}$ was superimposed over the main rf-field and applied between two opposite middle segments to selectively and simultaneously eject multiple ion species with different mass-to-charge ratios using resonance dipolar excitation waveforms [48].

Segmentation of the selection quadrupole rods was implemented to screen the fringing rf-fields during ion excitation and ejection [49]. When employing ion preselection, the dc-offset of the middle segment was pulsed to $5 \mathrm{~V}$ to create a shallow axial potential well in the center of the selection quadrupole assembly. During ion trapping in the "infinity" ICR cell [50], the middle segment bias was increased to $20 \mathrm{~V}$, efficiently quenching any ion species remaining in the selection quadrupole. Two apertures with diameters of $5 \mathrm{~mm}$ and 3.8 $\mathrm{mm}$ were positioned between the selection and accumulation quadrupoles for efficient ion focusing and trapping in the accumulation quadrupole. Another conductance limit with an aperture diameter of $3.8 \mathrm{~mm}$ was placed immediately downstream of the accumulation quadrupole to facilitate ion trapping and ejection from the quadrupole and to interface the quadrupole assembly with a differentially pumped commercial electrostatic ion guide. The pressure in the upstream region of the electrostatic ion guide was $4 \times 10^{-7}$ torr, and $7 \times$ $10^{-10}$ torr in the ICR cell. Ion trapping, cooling, and ejection from the accumulation quadrupole were accomplished by pulsing the dc-offsets of the accumulation quadrupole entrance/exit plates and the rods. Pulsed voltages were generated by a PC DAC board (National Instruments, Austin, TX) using software developed for this work, and then amplified by a built-inhouse 4-channel power amplifier. One of the channels of the power amplifier was used to vary a potential at the exit funnel plate. The timing for different events in the accumulation quadrupole (e.g., ion trapping) was determined by a TTL trigger generated by the Bruker FTICR data station. The accumulation quadrupole operated at a peak-to-peak rf-amplitude of $375 \mathrm{~V}$. A charge collector electrode was placed behind the ICR cell to monitor external ion trapping and transfer to the ICR cell. The charge collector was coupled to a current-tovoltage converter with a ratio of $1 \mathrm{nA} / \mathrm{V}$ that allowed detection of 1-ms pulses of ion current at amplitudes of less than $50 \mathrm{pA}$. A typical FTICR acquisition scan during capillary LC separation was $\sim 1.6 \mathrm{~s}$, including $0.5 \mathrm{~s}$ external ion accumulation, $0.1 \mathrm{~s}$ storage in the accumulation quadrupole, and $0.8 \mathrm{~s}$ detection (512 K datasets).

\section{Data-Dependent Acquisition}

The data-dependent external ion preselection was implemented with two alternating sequences [34]. Ions generated by the ESI source were non-selectively trapped in the accumulation quadrupole. Following a short storage period, the externally accumulated ions were ejected to the ICR cell and captured using gated trapping [51]. During the storage period used for collisional damping of ion kinetic energy and for modulation of ion potential energy in the accumulation quadrupole, the potential at the ion funnel exit plate was increased to $80 \mathrm{~V}$ so that no ions from the ESI could enter the quadrupole interface. During ion excitation in the ICR cell, a TTL trigger pulse from the Bruker data station was applied to the 12-bit ADC making it ready for data acquisition. Acquired mass spectra were converted to determine the corresponding secular frequency spectra (of ion oscillation) in the selection quadrupole and achieved by a superposition of excitation sine waveforms synthesized with our ICR-2LS software and generated by a $512 \mathrm{~K}$ plug-in PC DAC board. This was then immediately applied to the central segments of the selection quadrupole rods as an auxiliary rf-field. Using this approach one or several of the most abundant species detected at any $m / z$, but excluding the internal calibrants, could be effectively ejected from the selection quadrupole. This allowed external ion accumulation of lower abundant species over extended periods. To maintain a higher duty cycle, the auxiliary 
rf-field was switched off immediately following the ion transfer to the ICR cell, thus allowing for non-selective external ion trapping in the accumulation quadrupole to proceed simultaneously with analyzing the lower abundance ion species in the ICR cell.

\section{Sample Preparation}

D. radiodurans $\mathrm{R} 1$ was cultured in TYG media to stationary phase at $30{ }^{\circ} \mathrm{C}$ with shaking at $225 \mathrm{rpm}$ in normal and $>98 \%{ }^{15} \mathrm{~N}$ media (Martek Biosciences, Columbia, $\mathrm{MD})$. At $\mathrm{OD}_{600 \mathrm{~nm}}$ of 1.0 both cultures were combined and the cells harvested by centrifugation at 10,000 $\times g$ for $30 \mathrm{~s}$. The cells were resuspended in $200 \mu \mathrm{l}$ of PBS buffer and lysed by bead-beating in the presence of 0.1 $\mathrm{mm}$ acid zirconium beads for three $60 \mathrm{~s}$ cycles at 5000 $\mathrm{rpm}$. The samples were kept on ice for $60 \mathrm{~s}$ between each cycle. The cell lysate was recovered and centrifuged at $10,000 \times g$ for $10 \mathrm{~min}$ to remove any cell debris and then digested with trypsin. The lysate concentrations were $\sim 0.9 \mathrm{mg} / \mathrm{ml}$.

Direct infusion experiments were performed with peptides/proteins and electrospray calibrant solution [52] purchased from Sigma (Sigma Chemicals, St. Louis, MO) and Agilent (Palo Alto, CA), respectively, and used without further purification. The peptides/proteins were dissolved in a water:methanol:acetic acid solution (49:49:2 vol\%) at concentrations ranging from $0.1 \mathrm{mg} / \mathrm{mL}$ to $2 \mathrm{pg} / \mathrm{mL}$. The fluorocarbon polymer electrospray calibrant solution was diluted in acetonitrile to a concentration of $0.01 \mathrm{mg} / \mathrm{mL}$. The solutions were infused to the ESI source at a flow rate of 300 $\mathrm{nL} / \mathrm{min}$ using a syringe pump (Harvard, South Natick, MA).

\section{Capillary LC Instrumentation, Automation, and Methods}

Capillary LC system overview. The schematic for the liquid chromatography gradient and flow control system is shown in Figure $2 \mathrm{~b}$. This system was developed, designed, and fabricated in-house. The system was designed and tested for operation up to 10,000 psi. The basic system is an optimized, automated, and a more robust advanced version of a previously described system [26]. The goals for the LC and fluidics system were to simultaneously provide the following: (1) Very high performance, (2) very high pressure, (3) high levels of robustness and reliability, and (4) capabilities for high throughput and continuous, 24/7 operations. It was also designed to be flexible, easily modifiable, and extensible for future expansion of capabilities and improvements in basic technologies. All aspects of the LC system were directed at maintaining the highest chromatographic performance feasible. As such, all tubing, connectors, and valves were selected to minimize dead volumes and extra-column dispersions. The critical LC valves had 0.006 in. $(150 \mu \mathrm{m})$ thru-ports and improved port-to-port volumes. All critical connectors were newly developed low-volume units designed by Valco (Houston, TX) for high-performance micro- and nanoflow chromatography.

High pressure LC valves. The ChemInert units valves (Valco) were critical for both chromatographic performance and system reliability in continuous, $24 / 7$ operations. They are significantly improved versions of the positive feedback ChemInert valves, first described by Shen et al. [26] for use in high-efficiency LC-FTICR MS analyses of proteomics samples, incorporating an advanced polymer material in the rotor seal. This new material permits high-pressure operations $(10,000$ or 20,000 psi) and improved stability of the internal flow channel in the rotor seal, resulting in increased injection cycles and longer service life at high pressures. The system (Figure 2b) includes one 6-port valve (VINJ) (10) designed with one radial port, allowing it to accept the vertical injection port insert required to operate with the autosampler system described below. Three more 4-port valves were used in the core LC system: Solvent selection (VSS), Column Selection (VCC), and Mixer Purge (VMP). Although designed to operate with multiple columns, only one was initially implemented. Two more 4-port valves, VPA, (3) and VPB, (4), were used to facilitate and control the re-fill of the syringe pumps. These valves had larger thru-ports of 0.030 in. (760 $\mu \mathrm{m})$, because much larger flows were required and deadvolumes are not a factor at this point in the system. Refill was controlled via the PNNL Fluidics Control Software. Movement and actuation of all six valves was accomplished by a high-speed microelectronic actuator (Valco). The four actuators for the four core LC valves were grouped within the LC sub-chassis, thus minimizing valve-to-valve connection distances. Valve switching was directly controlled by the PNNL Fluidics Control Software, and coordinated with the rest of the system by the top-layer PNNL Automation Architecture (PAA) software.

LC Pump gradient control and generation. Two highpressure (10,000 psi) LC pumps (1) and (2) (model 100DM, Isco, Lincoln, NE) were used for mobile-phase delivery. The LC system is a constant-pressure type, providing greater robustness and immunity to microleaks. Gradient generation was performed via a mixer/ dilution chamber initially containing $2.5 \mathrm{~mL}$ of mobile phase A (MP-A). Over the gradient time, the mixer contents were diluted with mobile phase B (MP-B) in a controlled manner through a bypass column which is placed downstream of the mixer. Mobile phase was supplied to the analytical column at a mixer split point. In this system and these experiments, the total gradient flow through the mixer was $\sim 20 \mu \mathrm{L} / \mathrm{min}$. During the Mixer Purge period of the gradient cycle, MP-A was directed to the mixer via the valve $V_{m p}$. Flow during this phase was controlled by a high-flow by-pass column. The gradient used was as follows: Inject; Wash, 20 
min; Gradient: 0 to $60 \%$ B over 150 min; Purge Mixer to $100 \%$ A, 2 min; Re-equilibrate, $60 \mathrm{~min}$. The timing and synchronization of the gradient was controlled by the PNNL Fluidics Control Software.

Chromatography. The chromatography employed is a revered phase. The stationary phase is $\mathrm{C} 18$. The mobile phase is a water-acetonitrile gradient. Columns were packed as described by Shen et al. [26]. Stationary phase specifications for the analytical column were as follows. Packing material: Jupiter C-18, $5 \mu \mathrm{m}$ particles, $300 \AA$ pore size pore size, (Phenomenex, Terrence, CA). The column tubing was fused-silica capillary, $650 \mathrm{~mm}$ long $\times 150 \mu \mathrm{m}$ i.d. $\times 360 \mu \mathrm{m}$ o.d. (Polymicro Technologies, Phoenix, AZ). The low-flow bypass column (7) was packed with $5 \mu \mathrm{m}$ Jupiter C-18 particles. The column tubing was fused-silica capillary, $80 \mathrm{~mm}$ long $\times 150 \mu \mathrm{m}$ i.d. $\times 360 \mu \mathrm{m}$ o.d. (Polymicro Technologies, Phoenix, AZ). The high-flow bypass (mixer purge) column (8) was packed with $15 \mu \mathrm{m}$ Jupiter C-18. The column tubing was stainless steel HPLC tubing, $100 \mathrm{~mm}$ long $\times$ 0.030 in. $(750 \mu \mathrm{m})$ i.d. $\times 1 / 16$ in. o.d. (Valco, Houston, TX). Where needed, PEEK tubing (Valco, Houston, TX), $1 / 16$ in. o.d., and various i.d.s were used as sleeves to capture the silica capillaries in 1/16 in. HPLC fittings. Mobile Phase A was composed of water: acetic acid: trifluoroacetic acid (99.4:0.5:0.1 vol\%). Mobile Phase B was composed of water:acetonitrile: trifluoroacetic acid (79.9:20.0:0.1 vol\%). The water used in the experiments was produced in-house by a NANOpure water system (Barnstead/Thermolyne, Dubuque, IA). All other chemicals were HPLC grade and were purchased from Sigma Aldrich (Milwaukee, WI). All flows were as follows except as noted: Analytical column $2 \mu \mathrm{L} / \mathrm{min}$, flow split column $18 \mu \mathrm{L} / \mathrm{min}$. The LC peak capacities obtained based upon the peak widths of lower abundance species were generally $\sim 550$.

PAL autosampler. Sample loading and injection into the system and the LC column were performed by an HTS Model PAL autosampler (LEAP Technologies, Carrboro, NC). Custom injection cycle methods for the autosampler were written in Cycle Composer, the vendor-supplier control software and development environment. The PAA software running on the system control computer performed the actual control and synchronization of the autosampler with the other major components of the integrated LC/FTICR-MS system. Communication between the PAA software and the Cycle Composers software was implemented via COM/DCOM (Microsoft) technologies.

\section{System Automation Software}

The automation strategy for the overall LC-FTICR instrument was designed following a modular approach. From the software standpoint, the FTICR and capillary LC system with the PAL autosampler were independent and communicated with each other over the intra- net using Microsoft's distributed component object modeling (DCOM) protocols. A schematic of the automation architecture and an event sequence chart are shown in Figure 4. The Bruker FTICR data station running VB_Broker, XMASS 6.0, and Bserver programs, a Pentium IV PC to control the front-end ion optics elements, and Pentium III laptop for control of the LC and autosampler jointly managed control of the LCFTICR system. The stand-alone PC ran the developedin-house FTICR DC Control, Analog Output, and our ICR-2LS programs, which were used to generate dcpotentials for the FTICR front-end and interface, pulsed voltages at the accumulation quadrupole elements and the exit funnel plate, and to perform data-dependent ion preselection experiment, respectively. The laptop was associated with an LC/autosampler component to provide a fully automated module used to run both the commercial driver program for the PAL autosampler as well as the developed-in-house LC-MS control program that provided a sample table and determined the event sequence during LC separations. The software hierarchy constituted three different levels. The lower device communication level enabled DCOM communications over the intranet between LC-MS Control, VB_Broker, XMASS, PAL driver, and FTICR DC control programs that determined synchronization of sample handling, capillary LC separation, and FTICR data acquisition. The middle level incorporated the sample table with an editor. The experiment timing (e.g., the duration of separation or wash time) was preset at this level. The upper level provided a necessary interface and communication with our data management system (DMS) in an automated fashion. A typical automated LC-FTICR experiment included rinsing the sample injection syringe with an aqueous solution, loading a sample from an autosampler tray to a sample loop, and injecting the sample to an LC column followed by a 20 min wash, 150 min LC separation, 4 min purging of the mixer, and 60 min re-equilibration of the LC column. The event sequence chart in Figure $4 \mathrm{~b}$ shows the status of different components at the same time. For example, at the beginning of the sequence (time $=0$ ), solvent $\mathrm{A}$ is flowing through the column (see HPLC and VALVE, SOLVENT SELECT), injector valve is in "inject" position (see VALVE, SAMPLE INJECT), the autosampler is inactive (see AUTOSAMPLER), valve purging a mixer is in "off" position (see VALVE, MIXER PURGE), column is not selected (see COLUMN SELECT), and a mass spectrometer is "on hold" (see MS DATA DELAY and MS DATA ACQUISITION). During the inject event (see top of the graph and vertical dashed line), the autosampler is active, returning to its idle position after loading sample into a $10 \mu \mathrm{L}$ injection loop, SAMPLE INJECT VALVE is switching back to the "inject" position so that the solvent flow would bypass the injection loop and mass spectrometer receives a programmed delay command. A typical LC-FTICR run cycle was completed in about $3.9 \mathrm{~h}$ and resulted in acquisition of $\sim 5200$ mass spectra and $\sim 10 \mathrm{~GB}$ of raw data. Automa- 


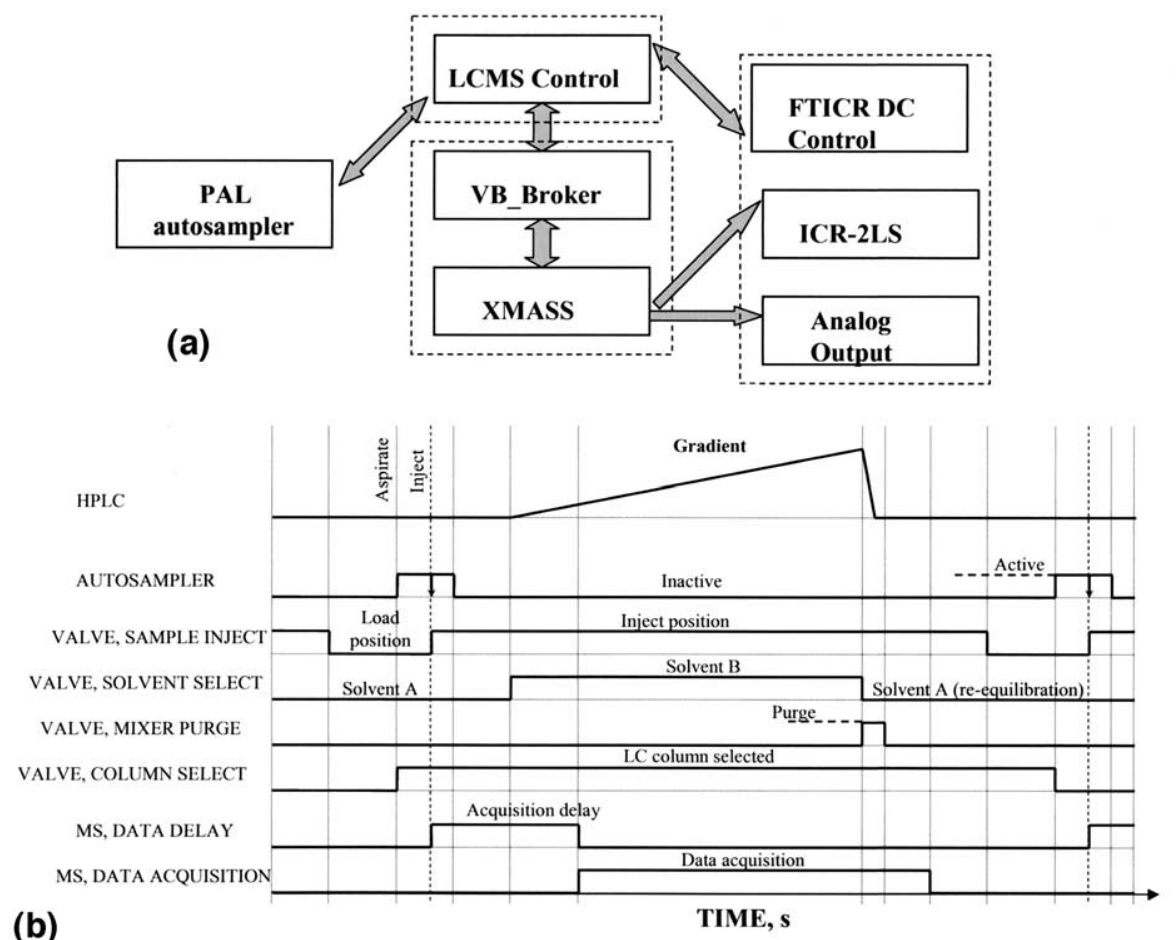

(b)

TIME, $\mathrm{s}$

Figure 4. (a) Diagram of the automation software architecture. "LC-MS Control" is run on the laptop, "FTICR DC Control", "Analog Output", and "ICR-2LS" programs are run on the stand-alone PC; "VB_Broker" and "XMASS" are run on the Bruker data station. The arrows represent COM/DCOM communication between different processes on three different computers showed schematically as three dashed boxes. (b) Sequence of events during capillary LC separation and FTICR data acquisition.

tion of the LC-FTICR system enabled $\sim 6$ runs per day and generating 50-60 GB of raw data.

\section{Results and Discussion}

The aim of this work was not only to implement a fully automated capillary LC-FTICR system, but also to do so in a fashion that provides very high performance of both the capillary LC and FTICR components. Thus, we extensively characterized and optimized system performance.

\section{Direct Infusion Experiments}

The FTICR mass spectrometer performance approaches ideal limits only if ions are located at or near the center of an ICR cell in both axial and radial directions and space charge effects are not excessive. In the cell center ions are least affected by electric or magnetic field inhomogeneities, and the coupling between radial motion and axial oscillations during excitation is minimized. Thus, it is desirable to "cool" ion translational motion so that ions axially reside near the ICR cell mid plane. Ion cooling becomes even more important when ions are generated externally and introduced into the ICR cell in pulses. When produced at an elevated pressure (e.g., from ESI emitters operating at atmospheric pressure), ions are generally introduced into an external (to the ICR cell) accumulation rf-only multipole through one or several stages of differential pumping, typically incorporating rf-only ion guides (e.g., quadrupoles). To minimize ion losses and assist ion transmission through the fringing rf-fields of the rf-only ion guides, accelerating and focusing dc-potentials from a few tenths to several volts are typically applied to the rf-only ion guides and conductance limits. The kinetic energy distribution is primarily a result of collisions that occur in the lower pressure regions and where electric fields are used to accelerate ions to higher kinetic energies. Therefore, the total energy (i.e., the sum of the kinetic and potential energies) of, for example, a cloud of the singly charged ions trapped in the accumulation rf-only multipole would be in the range of few $\mathrm{eV}$. Injecting this ion cloud into the ICR cell requires the use of trapping potentials in the range of a few volts and one would ideally like to reduce this to a few tenths of a volt prior to ion excitation/detection to achieve high resolution. If ion excitation/detection immediately follows ion capture in the ICR cell (i.e., without an ion cooling event), the acquired FTICR signal exhibits reduced mass resolution, mass accuracy, peak intensity and distorted peak shape due to a broader ion spatial and energy distribution.

The commercial FTICR instrument was initially equipped with a hexapole for external ion accumulation, and typically uses two approaches for trapping 
ions in the ICR cell. The first and most commonly used approach is that of Caravatti [54] in which ion translational energy is reduced because of lateral deflection (i.e., "side-kick") of the ion beam entering the ICR cell. This causes a second motion component in a direction perpendicular to the magnetic field to be imparted to ions such that the magnitude of the vector sum of the two ion velocity components remains the same. In essence, side-kick succeeds in trapping externally injected ions by converting axial energy into cyclotron energy with a significant increase in the ion cloud magnetron radius, a disadvantage of the approach. Another drawback is that ion deflection in the dcelectric field is mass-to-charge dependent.

The second approach is based on gated trapping. In this approach the front trapping plate is grounded during ion injection to the ICR cell and then rapidly reinstated to establish an axial potential well, so that the ions have insufficient kinetic energy to escape from the cell. A pulsed introduction of a collision gas is commonly used to reduce (i.e., cool) the initially excessive axial energy of ions. Such cooling also reduces the ion cyclotron radius, but, at the same time, facilitates magnetron expansion. In addition, pulsing collision gas into the ICR trap significantly decreases the duty cycle (spectrum acquisition rate) of the FTICR instrument if high performance is to be maintained. Other ion cooling approaches include ion evaporative [55], adiabatic [36, 38], resistive [56] and sympathetic [57] cooling. Though these approaches have been shown to provide effective cooling for particular applications, they have not been widely adopted because of a decrease in signal intensity (e.g., evaporative cooling), reduced capability of ion cooling over a broad $\mathrm{m} / \mathrm{z}$ range (e.g., adiabatic cooling), inefficiency at higher cyclotron frequencies where detection is mostly capacitive (e.g., resistive cooling) or extended cooling time for hot heavier ions to be cooled by electrons (e.g., sympathetic cooling). One of the objectives of this work was to develop a method for accumulating ions in the ICR cell that would eliminate the ion-cooling step and minimize ion axial oscillations without increasing magnetron radii. Implementation of this approach would then be expected to increase the duty cycle, mass measurement accuracy (MMA), and the resolution of LC-FTICR.

Figure 5a shows ion transmission of the modified FTICR instrument operating in the continuous mode. Micro electrospray-generated ions from a $10^{-6} \mathrm{M}$ solution of bovine ubiquitin were injected into the mass spectrometer at a flow rate of $100 \mathrm{~nL} / \mathrm{min}$ and then transmitted through the ion funnel/quadrupole interface, electrostatic ion guide, and ICR cell with entrance/ exit aperture diameters of $2 \mathrm{~mm}$ to impinge the charge collector coupled to an electrometer (Keithley Instruments, Cleveland, $\mathrm{OH})$. Figure $5 \mathrm{~b}$ and $\mathrm{c}$ shows the pulsed signal at the charge collector from the same sample detected after trapping ubiquitin ions in the accumulation quadrupole for $100 \mathrm{~ms}$. The voltage change during the ion ejection pulse can be understood
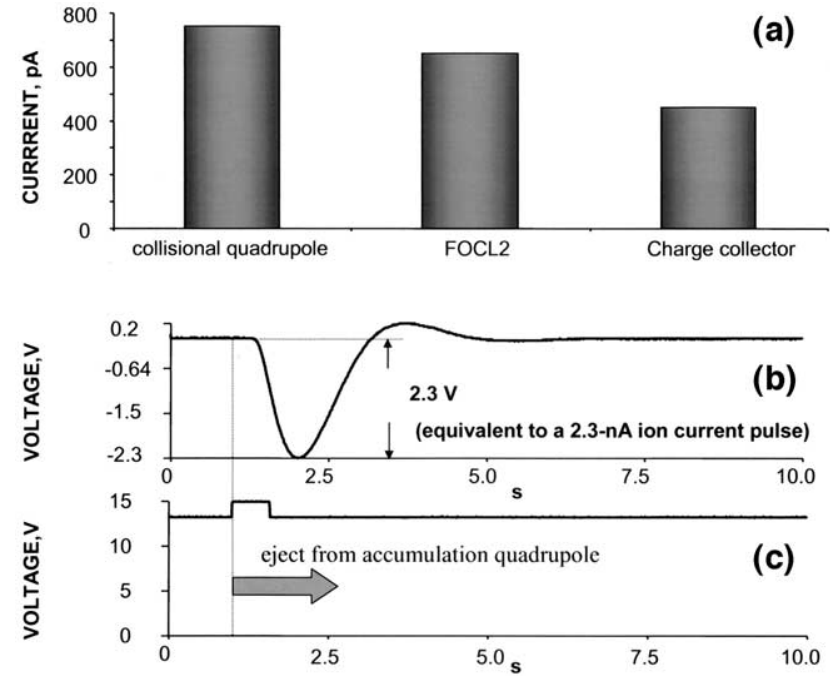

Figure 5. (a) Histogram of total ion current from a $10^{-6} \mathrm{M}$ solution of bovine ubiquitin measured at different elements of the FTICR instrument. (b) Total ion current pulse from a $10^{-6} \mathrm{M}$ solution of bovine ubiquitin detected at the charge collector. Ion accumulation time in the quadrupole was $100 \mathrm{~ms}$. (c) Trigger pulse indicating ion ejection from the accumulation quadrupole.

by comparing Figure 3 and Figure 5b. The rising edge of the trigger pulse in Figure 5b corresponds to the falling edge of the pulse applied to the Accumulation quadrupole exit plate (14) in Figure 3. A decrease in this potential enabled the ion ejection event from the accumulation quadrupole. The potentials at the accumulation quadrupole bias (13) and the accumulation quadrupole entrance plate (12) stayed at -2 and $+17 \mathrm{~V}$, respectively, for another $450 \mu \mathrm{s}$ corresponding to the average flight time of ions from the accumulation quadrupole to the charge collector (or FTICR cell). Following ion ejection from the accumulation quadrupole, the quadrupole bias was increased to $+10 \mathrm{~V}$ for $200 \mathrm{~ms}$ to purge any remaining ion population and prepare the trap for the next accumulation cycle. The pulsed ion current amplitude was 4-fold higher than that of the continuous ion beam. Given an entrance current of $\sim 700 \mathrm{pA}$ in the accumulation quadrupole, an ion accumulation time of $100 \mathrm{~ms}$ in the quadrupole and similar transmission efficiency for a packet of externally trapped ions as the continuous ion current, one would expect a total charge of $\sim 40 \mathrm{pC}$ be transmitted to the ICR cell. Integration of the ion current pulse striking the charge collector resulted in $\sim 4 \mathrm{pC}$, indicating $\sim 10 \%$ trapping efficiency in the accumulation quadrupole.

Figure 6 shows the time-of-flight (TOF) distributions of singly charged polymer ions with $\mathrm{m} / \mathrm{z}$ ranging from 500 to 2200 Da obtained with both the commercial (Figure 6a) and modified (Figure 6b) front-end interfaces. Note that since TOF distributions depend on $\mathrm{m} / \mathrm{z}$, the comparison was done in the same $\mathrm{m} / \mathrm{z}$ range. The externally accumulated ions were ejected and then trapped in the ICR cell using gated trapping followed by ion excitation and detection. Trapping was per- 

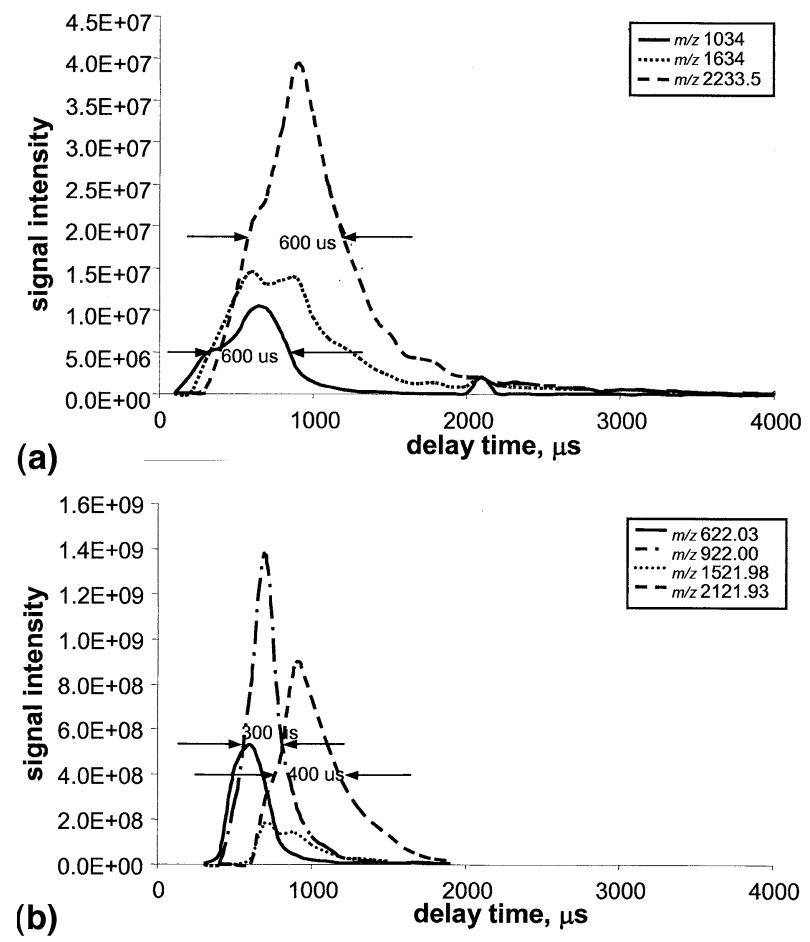

Figure 6. Time-of-flight distributions of externally trapped ions transmitted to the ICR cell using (a) commercial and (b) modified front-end interfaces.

formed using a simultaneous increase in the potentials at the front and back trapping plates. Due to higher transmission efficiency of the inlet capillary/ion funnel/quadrupole assembly than the inlet capillary/ skimmer/hexapole arrangement, typically 5- to 10-fold shorter ion accumulation times were used with the ion funnel/quadrupole interface to obtain similar peak intensities. The TOF distributions were derived by varying the delay between the drop in potential at the accumulation multipole exit aperture, resulting in ion ejection from the multipole and a rise in potentials at the front and back trapping plates of the ICR cell used to establish an axial potential well and to capture ions. A typical full-width at half-magnitude (FWHM) for the TOF distributions of ions ejected from the quadrupole operating at a pressure of $10^{-5}$ torr was significantly improved ( $\sim 2$-fold less) compared with that obtained using the commercial hexapole functioning at a pressure of $\sim 10^{-2}$ torr. The period of ion axial oscillation in the cylindrical cell can be estimated as follows [53]:

$$
T_{\text {axial }}=2 \pi \sqrt{\frac{m a^{2}}{2 z e V_{t r} \beta}}
$$

where $a$ is the length of the ICR cell, $m$ is the ion mass, $\beta=2.84$ is the geometrical factor of the cylindrical cell, $V_{t r}$ is the trapping voltage, $z$ is the charge state, and $e$ is the elementary charge. For the "infinity" ICR cell with a length of $60 \mathrm{~mm}, T_{\text {axial }}$ is $\sim 720 \mu$ s at $V_{t r}=0.5 \mathrm{~V}$ and $\mathrm{m} / \mathrm{z}$ $=1000 \mathrm{Da}$. Hence, the efficiency of gated trapping is expected to approach $100 \%$ for a delay time corresponding to the maximum of the TOF distributions for ions ejected from the accumulation quadrupole. Note that further narrowing of TOF distributions would result in a decrease in intensities for ions at the extremes of the detected $\mathrm{m} / \mathrm{z}$ range compared with the ion signal corresponding to the middle of the $\mathrm{m} / \mathrm{z}$ range. This is an undesirable consequence of TOF discrimination in the electrostatic ion guide and that effectively limits efficiency for higher and lower $\mathrm{m} / \mathrm{z}$ ends of mass spectra. Therefore, we did not attempt to further expedite ion ejection from the $50 \mathrm{~mm}$-long accumulation quadrupole, for example, by segmenting the quadrupole rods [23].

Figures 7 and 8 demonstrate typical mass resolution obtained with broadband excitation and MMA routinely available with the ion funnel/quadrupole interface in direct infusion experiments using $10^{-6} \mathrm{M}$ solutions of bovine ubiquitin and electrospray calibrant mixture, respectively. This mass resolution and accuracy is characteristic of a novel method of ion introduction and accumulation in the ICR cell developed in this work. No ion-cooling step was used prior to ion excitation and detection. Following ion trapping in the accumulation quadrupole, the dc-bias of the quadrupole was rapidly reduced to a negative voltage (e.g., -2 V). Since typical ion trapping time in the accumulation quadrupole was comparable to or greater than the period required to reduce the translation energy of ions colliding with a buffer gas, the trapped ion cloud had a thermal energy distribution effectively superimposed upon the energy distribution from space charge. The latter energy distribution had a minimum on the quadrupole axis, reaching a maximum near the quadrupole rods. The instantaneous lowering of the accumulation quadrupole dc-bias did not change the thermal energy distribution for the majority of the trapped ions on the accumulation quadrupole axis. After a short delay of 50 $\mathrm{ms}$, ions were ejected from the accumulation quadrupole towards the ICR cell. Since ions were ejected at a negative potential energy, the ion guide, the front trapping plate of the ICR cell and the side-kick electrodes were kept at negative potentials. No lateral ion deflection (i.e., side-kick) was used during ion introduction into the ICR cell. The rear trapping plate was maintained at a low positive potential of $0.5 \mathrm{~V}$ to provide ground potential in the middle of the ICR cell. Because the ions were ejected from the accumulation quadrupole at a negative dc-bias, they decelerated upon approaching the ground potential in the middle of the ICR cell. Following a short delay corresponding to the maximum of TOF distributions in Figure 6b, a potential at the front trapping plate of the ICR cell was rapidly increased to a positive voltage of $0.5 \mathrm{~V}$ while keeping the potential of the side-kick plates at $-2 \mathrm{~V}$. This created a shallow well $(\sim 0.2 \mathrm{~V})$ in the middle of the ICR cell where ions were trapped. Ions with kinetic energies above $0.2 \mathrm{~V} \times$ charge should escape from this shallow well and be lost. 


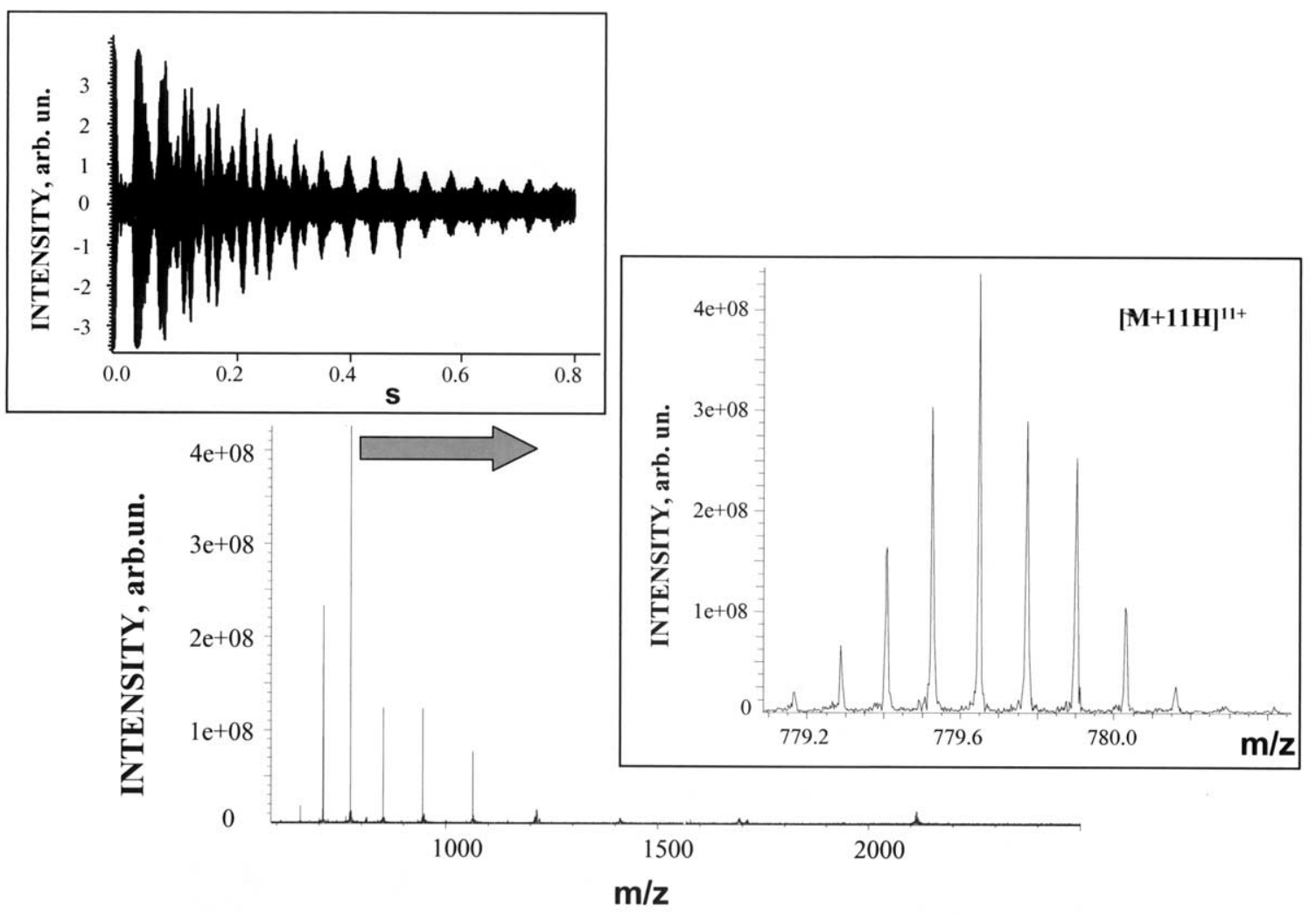

Figure 7. Typical transient signal and mass spectrum with a $10^{-6} \mathrm{M}$ solution of bovine ubiquitin acquired at a trapping potential of $0.5 \mathrm{~V}$ in the ICR cell.

This new ion trapping technique with ion energy modulation in the accumulation quadrupole is characterized by several important features. First, ion detection is virtually magnetron-motion free (i.e., the detected cyclotron frequency will be altered to a very small extent because of the low magnetron component) since the non-homogeneity of the electric field from the

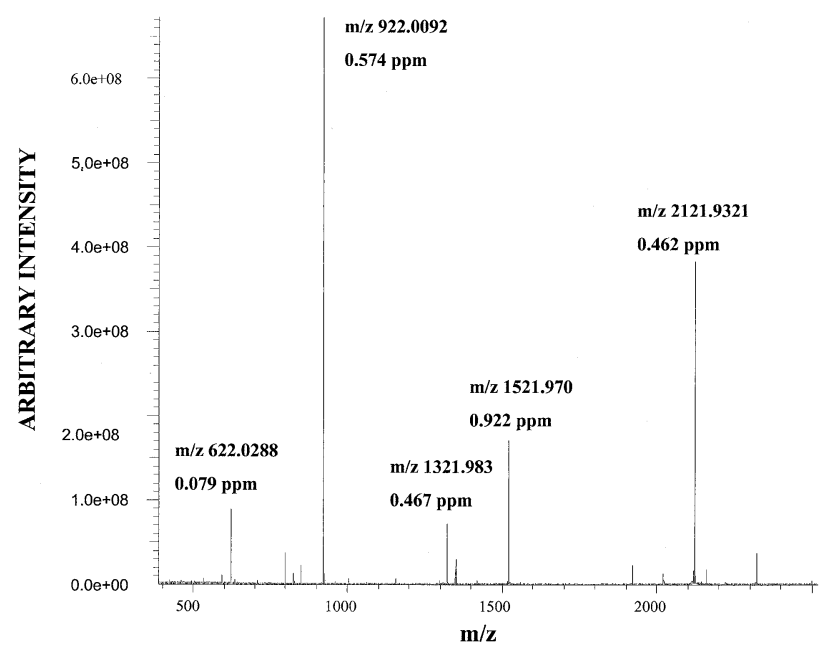

Figure 8. Typical mass accuracy in FTICR acquisition of a mass spectrum from a $10^{-7} \mathrm{M}$ electrospray calibrant solution using 0.5 $\mathrm{V}$ modulated-energy gated trapping in the ICR cell. Ion accumulation time was $200 \mathrm{~ms}$. trapping plates is minimized in the middle of the ICR trap. Second, a narrow energy distribution of the ions entering the ICR trap $(<0.2 \mathrm{eV})$ results in high-efficiency trapping in a shallow well of $0.2 \mathrm{~V}$, thereby increasing instrument sensitivity. Third, a trapped ion cloud does not need to be cooled in the ICR cell since reduction of the ion translational energy distribution is already performed in the accumulation quadrupole at an elevated pressure. The maximum kinetic energy of ions trapped in the ICR cell is determined by the dc-bias of the accumulation multipole. Avoiding the cooling event in the ICR cell also significantly improves the spectrum acquisition rate. Fourth, the coupling of ion motions in radial and axial directions during ion excitation is minimized since imperfections of the excitation field in close proximity to the trapping plates does not affect ions trapped in the middle of the ICR trap. This feature is less important for the linearized "infinity" cell, but becomes significant for conventional (e.g., a cylindrical) cell designs.

Figure 9 shows automated data-dependent ejection of the most abundant species using resonant dipolar excitation in the selection quadrupole. The polymer ion species at $\mathrm{m} / \mathrm{z} 922.009$ were identified in the broadband accumulation mass spectrum as the most abundant ion species and were selectively ejected in the immediately following acquisition with a resolution of $\sim 100$. Ion ejection was performed at a Mathieu parameter $q$ of 

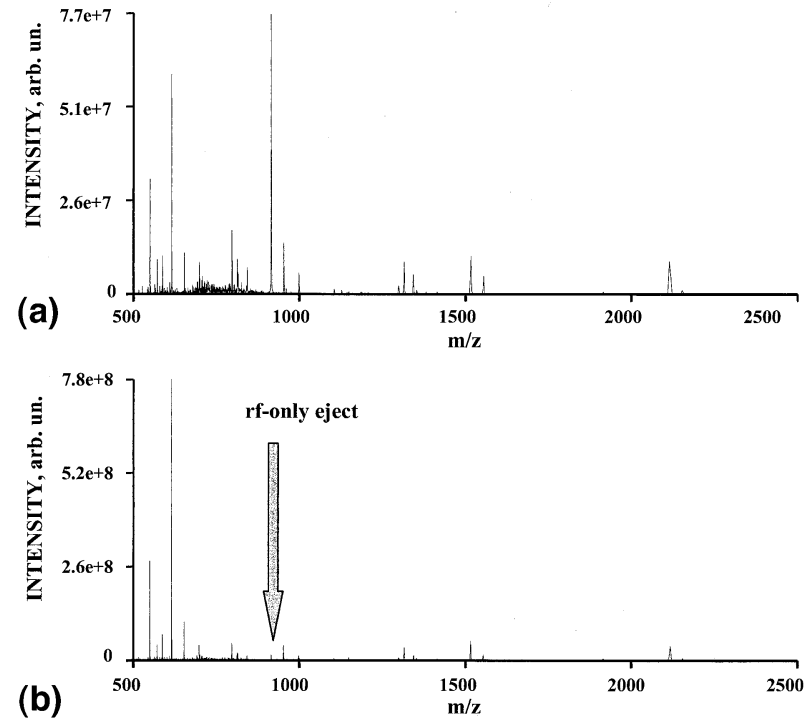
$\mathbf{m} / \mathbf{z}$

Figure 9. Data-dependent selective ion ejection experiments: (a) Broadband accumulation of a $10^{-7}$ electrospray calibrant solution at an accumulation time of $50 \mathrm{~ms}$, (b) selective ejection mass spectrum of the same solution at an accumulation time of $250 \mathrm{~ms}$. The most abundant peak at $\mathrm{m} / \mathrm{z} 922.009$ was data-dependently ejected in the selection quadrupole using rf-only resonant excitation.

$\sim 0.4$ and ion accumulation time was increased by a factor of 5 . An axial potential well of $\sim 1 \mathrm{~V}$ was formed in the middle section of the selection quadrupole during excitation. To quench any remaining ion populations in this region of the selection quadrupole, the potential at the middle segments was increased to $20 \mathrm{~V}$ during ion trapping in the ICR cell. This selective ion ejection approach enables the DREAMS capability that has been previously shown to enhance the dynamic range of FTICR measurements [34, 35, 49].

\section{Capillary LC-FTICR Analyses}

FTICR and LC-FTICR have not generally been regarded as robust technologies and have often been considered unsuitable for the high-throughput measurements (e.g., in proteomics). The unattended operation of a capillary LC-FTICR system posed major challenges associated with controlling trapped ion populations used for measurements and other related mass calibration issues.

Figure 10 illustrates the reproducibility of automated capillary LC-FTICR analyses employing the dual-channel electrodynamic ion funnel with internal calibration. Total ion current chromatograms shown in Figure 10 were obtained for three unattended overnight "back-toback" runs with a $D$. radiodurans proteome sample. The chromatographic peaks correlated to within 1-2\% (even without normalization of elution times), demonstrating the high reproducibility of peptide elution time with automated injection/re-equilibration of the capillary LC system. Figure 11 compares mass spectra from two separate capillary LC-FTICR runs conducted two weeks

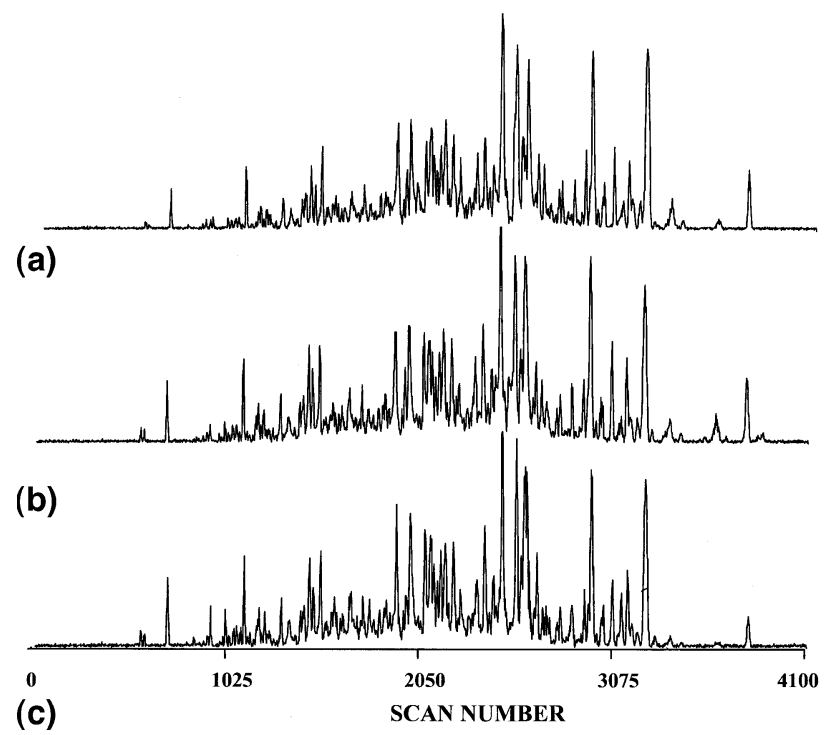

Figure 10. Total ion current chromatograms of the three "backto-back" overnight unattended LC-FTICR runs with a $0.1 \mathrm{mg} / \mathrm{mL}$ bacterial (D. radiodurans) proteome sample (DR102). Each separation included a $20 \mathrm{~min}$ wash, $150 \mathrm{~min}$ LC separation, $2 \mathrm{~min}$ purging of the mixer, and $60 \mathrm{~min}$ re-equilibration of the LC column. The total time of this automated experiment was $\sim 9 \mathrm{~h}$.

apart for a tryptic digest of ${ }^{14} \mathrm{~N} /{ }^{15} \mathrm{~N}$ labeled proteins from the same aliquot of a different $D$. radiodurans sample. Regions of mass spectra acquired at the same elution time (i.e., at the same scan numbers) demonstrate that identical peptides are detected having similar peak intensities ( $\sim 10$ to $50 \%$ absolute variation). The mass spectral peaks at $\mathrm{m} / \mathrm{z} 815.45$ and 738.42 were identified as R.SAVEEGIVAGGGTTLLR.V (tryptic fragment DR607.t58) and R.APLSVYGEVNTGLR.A (tryptic fragment DR0673.t11) peptides from DR607 GroEL and DR0673 hypothetical protein, respectively. Figure 12 depicts the integrated signals over the elution times (i.e., peaks from TIC chromatograms) of ${ }^{14} \mathrm{~N} /{ }^{15} \mathrm{~N}$ labeled peptides shown in Figure 11. The area under the peaks in Figure 12 provide information on the abundance of a particular peptide detected in the course of the capillary LC separation. Deviations in abundances of the same peptides detected two weeks apart were found to be $<23 \%$ (Figure 12a and $b$ ) and $<13 \%$ (Figure $12 \mathrm{c}$ and $\mathrm{d}$ ) of elution time variations (corresponding to the maxima of the LC peaks) of 0.25 and $0.15 \%$, respectively. This illustrates the high stability for automated the capillary LC-FTICR instrument when operating continuously (24/7) over an extended period of time (e.g., a span of several weeks).

A major emphasis in the development of the automated capillary LC/FTICR instrument was to achieve high MMA for identification of enzymatically digested proteins. The utility of high MMA is particularly beneficial for the aforementioned AMT tag approach developed at our laboratory. In the initial demonstration of this approach [44] with Deinococcus radiodurans, only $\sim 70 \%$ of peptides identified with SEQUEST scores 

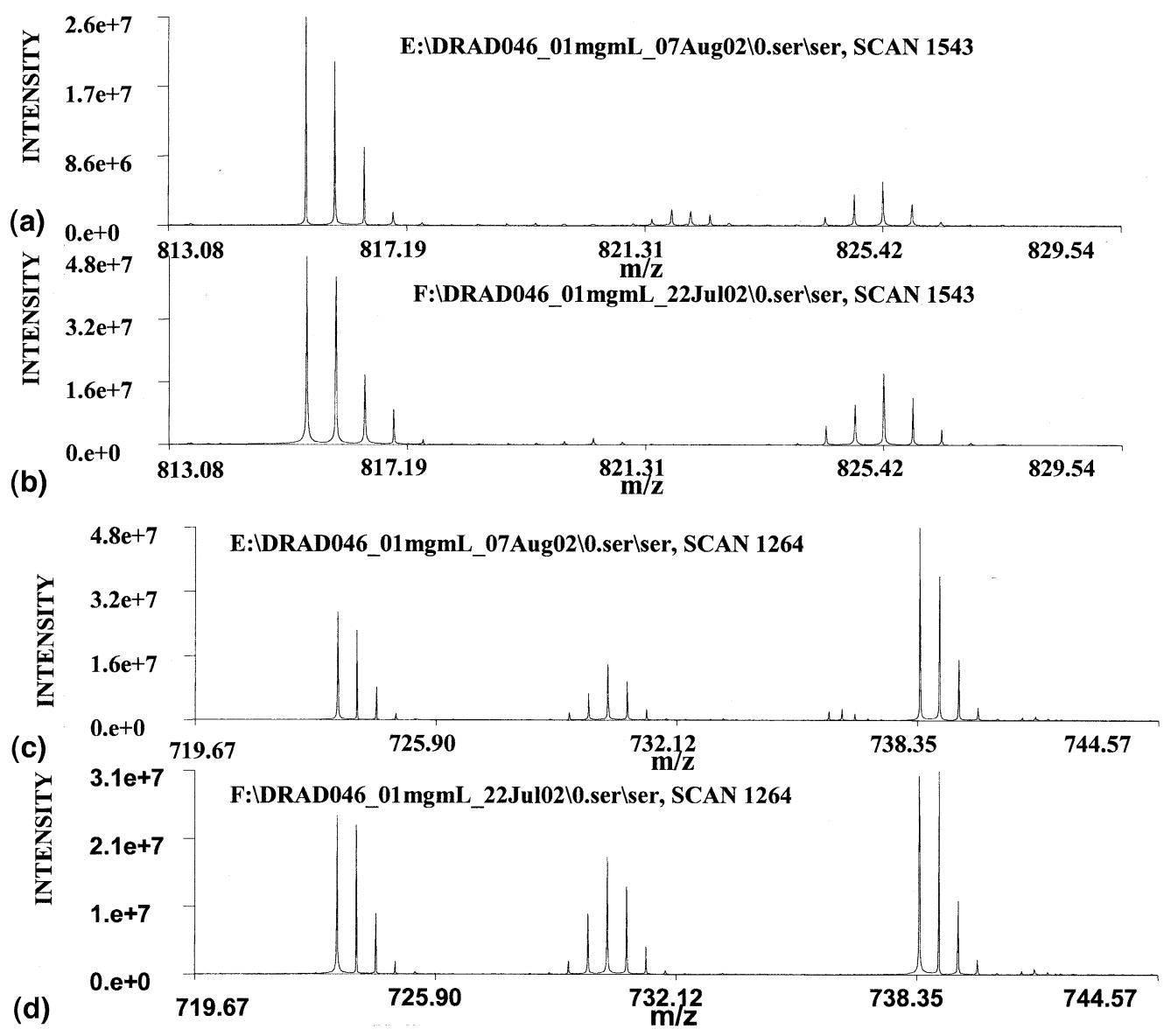

Figure 11. Portions of the mass spectra from two LC-FTICR runs conducted two weeks apart (July 22, 2002 and August 7, 2002) with samples from the same aliquot of a $0.1 \mathrm{mg} / \mathrm{mL}$ bacterial (D. radiodurans) proteome (DRAD046). The mass spectra in $\mathbf{a}$ and $\mathbf{b}$ and $\mathbf{c}$ and $\mathbf{d}$ correspond to the same elution times and the same $\mathrm{m} / \mathrm{z}$ ranges, respectively. Differences can arise due to a combination of changes in both LC and FTICR performance, as well as to the sample during storage.

above 2.0 were validated as AMT tags. An analysis of the conversion of PMT tags to AMT tags shows that almost all PMT tags having a SEQUEST cross correlation $\left(\mathrm{X}_{\text {corr }}\right)$ scores $>4.0$ were converted to AMT tags. A rapid decrease in the conversion of PMT tags to AMT tags, however, was observed for PMT tags identified with an $X_{\text {corr }}$ value less than 3.0, to $<40 \%$ for SEQUEST $X_{\text {corr }}$ scores of 2.0. This result illustrates the decreased reliability of peptide identifications for intermediate scores. Such identifications account for the majority of peptides identified in MS/MS experiments with ion traps in these studies. This highlights the advantage of using the high mass accuracy "filter" provided by FTICR to improve the overall peptide identification process by greatly reducing incorrect assignments for the large set of low to moderate confidence identifications.

As mentioned earlier, large variations in the intensities and composition of tryptic peptides in the course of capillary LC separation result in significant quantitative (i.e., "global" space charge) and qualitative (i.e., "local" space charge) changes of ion populations trapped in the ICR cell, leading to pronounced shifts of the detected cyclotron frequencies. Therefore, a set of internal reference masses (i.e., calibrants) is generally required to correct for the space charge related frequency shifts [58] and to achieve the highest accuracy. Note that the commercial Bruker FTICR instrument provides internal calibration by mixing calibrant and analyte ions in the ESI emitter. However, the practical utility of this approach is limited for two reasons. First, analytes/ calibrants can cause ionization suppression of co-eluting components from the complex proteolytic digests. Second, external accumulation of fixed calibrant levels and analytes for the same period reduces the effective dynamic range of capillary LC-FTICR measurements when analyte concentrations are low since accumulation of the lower abundance analytes is then limited by the presence of higher abundance internal calibrants.

The modified front-end interface provided independent ionization and introduction of analyte and calibrant species attributable to the use of two ESI emitters and separate heated inlet capillaries along with the independent control over the transmission of the calibrant and analyte species using the dual-channel electrodynamic ion funnel. In a typical LC/FTICR run both 

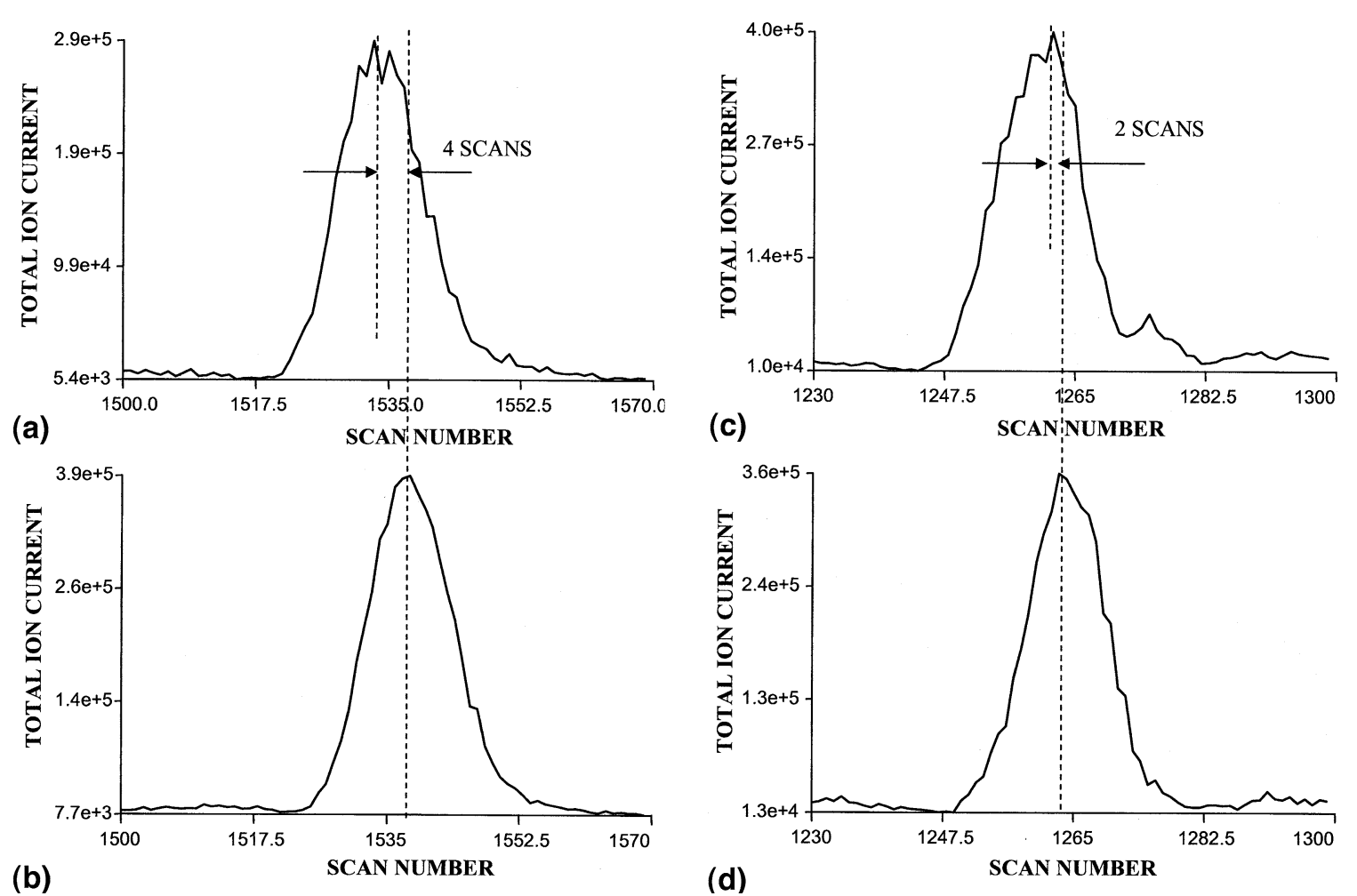

Figure 12. Elution profiles of R.SAVEEGIVAGGGTTLLR.V and R.APLSVYGEVNTGLR.A peptides at $\mathrm{m} / \mathrm{z} 815.45$ and 738.42 in Figure 11. The maxima of the elution peaks of the peptides detected on August 7, 2002 are offset by 4 scans (scan number 1543) and 2 scans (scan number 1265) relative to those detected on July 22,2002 , indicating reproducibility of the elution times of better than $0.5 \%$. The integrals of elution peaks correlate within $22 \%$ (a and $\mathbf{b}$ ) and $13 \%$ (c and $\mathbf{d})$. Systematic differences in elution times are eliminated in data analysis by the normalization of elution times using commonly observed peptides (effective internal elution time calibrants).

the analytes and calibrants were transmitted to the accumulation quadrupole for $150 \mathrm{~ms}$ followed by 450 ms accumulation of only the analyte species. Because of the presence of internal calibrants in each acquired mass spectrum, both external and internal calibrations were used for comparison. External calibration was performed using the widely adopted calibration equation [59]:

$$
m / z=A / f+B / f^{2}+C
$$

where $f$ is the detected cyclotron frequency, and $A, B$, and $C$ are the calibration coefficients. One particular set of calibration coefficients was used for external calibration of all mass spectra acquired in the course of LC separations. The presence of the calibrant peaks with known mass-to-charge ratios simplified the calibration procedure, making it less dependent on the instrument parameters (i.e., there is no need to calibrate the FTICR mass spectrometer before each LC run since frequency shifts of the calibrant peaks account for minor changes in the instrumental parameters). However, due to the space charge caused frequency shifts of calibrant ions, the MMA obtained with external calibration depends on the particular spectrum or spectra used for the calibration. Note that the intensities of the calibrant peaks decrease somewhat during the elution of the LC peptides and increase again at the end of the LC separation for reasons that are presently the subject of additional investigation. We found that better external calibration was obtained using spectra from the middle of the LC separation and where calibrant ions were most affected by the eluting peptides.

Internal calibration was performed using eq 2 after all calibration coefficients were fit using a LevenbergMarquardt algorithm [60] for each mass spectrum acquired during the LC separation. The frequencies of calibrant ions from adjacent scans were used with weight coefficients to provide higher statistical confidence. This enabled us to calibrate each mass spectrum after data acquisition. As an example, Figure 13 shows the deviation of errors for the peptide LVTDLTK from a $0.1 \mathrm{mg} / \mathrm{mL}$ tryptic digest of bovine serum albumin (BSA) obtained using external and internal calibration. Importantly, when comparing the peptide intensity profile and error distribution obtained with external calibration, one would notice that dispersion of errors significantly reduces upon achieving a certain intensity threshold. For example, the errors at the tails of the elution profile (scans $875-878$ and 900-905) increase 


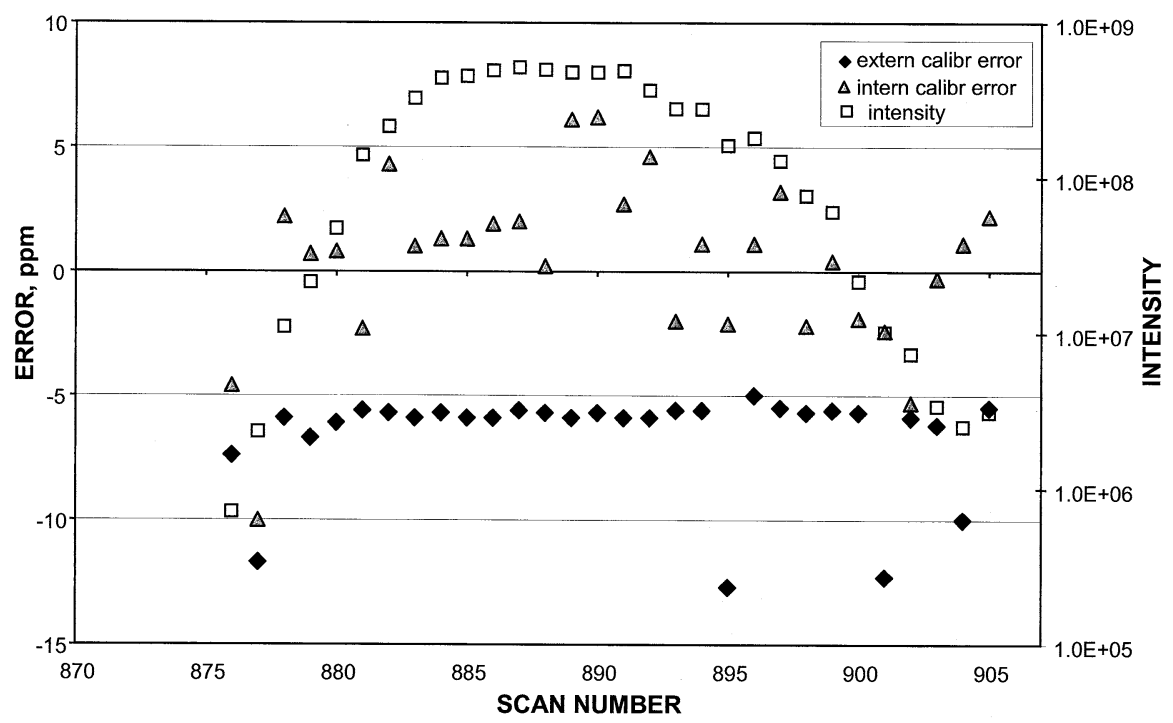

Figure 13. Distribution of errors obtained using external and internal calibration (using all calibrant peaks) and the dependence of the intensity on the scan number for the LVTDLTK tryptic peptide from a $0.1 \mathrm{mg} / \mathrm{mL}$ bovine serum albumin digest.

compared with those corresponding to the middle of the LC peak. We found this to be a general trend for both peptides and calibrants, which by far determines the mass accuracy with internal calibration. Since the calibration function in eq 2 is non-linear, a $0.2 \mathrm{mg} / \mathrm{mL}$ solution of leucine enkephalin was added to the electrospray calibrant solution to provide an additional reference peak at $\mathrm{m} / \mathrm{z}$ of 556.2754 in the lower $\mathrm{m} / \mathrm{z}$ end of a mass spectrum. All of the calibrant peaks in Figure 8 and leucine enkephalin were used for internal calibration of LVTDLTK. A reduction in the mass measurement error averaged over the number of scans was observed for the internally calibrated LVTDLTK peptide, although surprisingly the standard deviation of mass measurement errors increased. To determine the origin of this increase, we analyzed the frequency shifts of the observed peptides from a BSA digest due to application of internal calibrant peaks during LC separation. Roughly, 2600 "hits" were observed for possible BSA peptides within each run. A "hit" is defined as the observation of a possible BSA peptide during one FTICR scan. Many abundant peptides were observed 20 to 30 times over their LC peak width within the run. Figure 14 shows the histograms of peptide "hit" counts for the externally (Figure 14a) and internally calibrated (Figure 14b) $\mathrm{m} / \mathrm{z} 922.009 \mathrm{Da}$, as well as the externally calibrated $\mathrm{m} / \mathrm{z} 1321.932 \mathrm{Da}$ (Figure 14c). Each histogram bar corresponds to the number of matches within a 1-ppm bin. A shift of error distribution to $-10 \mathrm{ppm}$ in Figure 14a is due to the particular set of calibration coefficients used for external calibration. Since calibrant peaks were present in every mass spectrum during the LC separation, the option exists to externally recalibrate the entire LC run using calibrants from any mass spectrum. (The overall approach for mass calibration remains to be fully optimized; as mentioned above, we found that external calibration based on mass spectra from the middle of LC separation generally resulted in better accuracy. However, it is clear the flexibility for selection and use of multiple calibration modes, combined with the capability for controlling ion population, provides a basis for overall optimization.) A striking difference in the cyclotron frequency shifts was observed between using lower $(\mathrm{m} / \mathrm{z} 1321.932$, Figure 14c) and higher $(\mathrm{m} / \mathrm{z}$ 922.009, Figure 14a) abundance calibrant peaks. This result indicates that below a certain intensity threshold, the detected ion species experienced poorly predictable cyclotron frequency shifts (Figure 14c), which caused increased mass measurement errors. If lower intensity calibrants are included in the calibration, their errors propagate through the calibration equation (see the internal calibration data in Figure 13 and Figure 14b), resulting in much larger errors for both calibrants and peptides. To obviate this issue, only higher abundance calibrant peaks were used for further internal calibration. Figure 15 shows the distribution of mass measurement errors for the singly and doubly charged QTALVELLK peptide that was obtained using external and internal calibrations based on higher intensity calibrant peaks. As a result, both the average and dispersion of mass measurement errors improved for the internally calibrated data. Note that a single mass calibrant would not correct for the frequency shifts of singly and doubly charged ions of the QTALVELLK peptide, since the frequencies shifted in opposite directions.

Figure 16 shows the distribution of errors for 59 tryptic peptides from the BSA digest along with peptide intensities (Figure 16a) and the number of measurements leading to identification of a particular peptide (Figure 16b). Each data point in Figure 16a (error axis) shows the average mass measurement error 


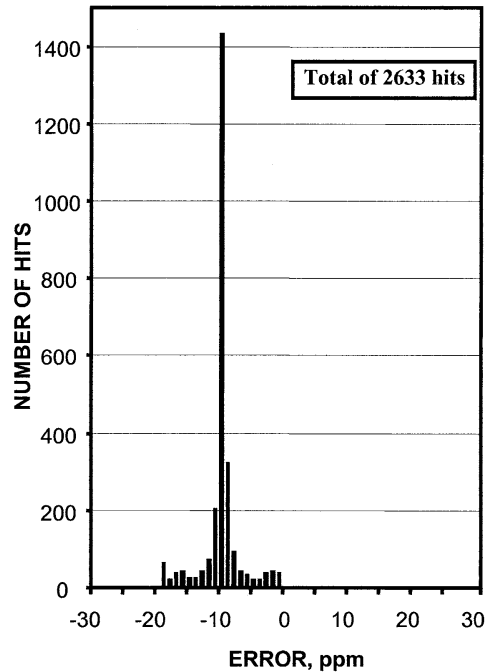

(a)

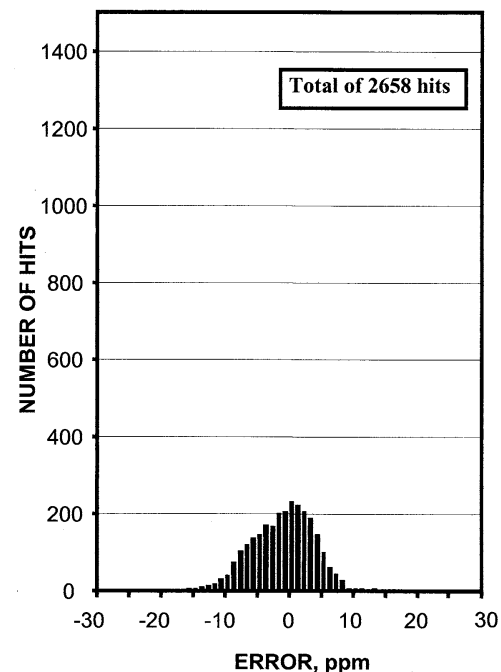

(b)

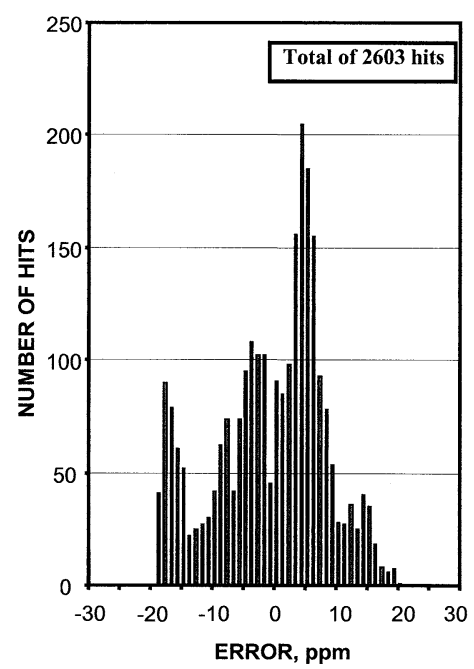

(c)

Figure 14. Dependence of the number of tentative identification "hits" on the mass measurement errors in FTICR detection of calibrant ions in the course of capillary LC separation using a $0.1 \mathrm{mg} / \mathrm{mL}$ bovine serum albumin digest. (a) $\mathrm{m} / \mathrm{z}$ 922.009, external calibration, (b) $\mathrm{m} / \mathrm{z} 922.009$, internal calibration using all calibrant peaks, (c) $\mathrm{m} / \mathrm{z}$ 1321.91, external calibration. Each bar corresponds to the number of hits within 1-ppm bin.

while the error bars correspond to the standard deviation of errors in identification of tryptic peptides over their elution time. Each bar in Figure 12b indicates the number of measurements that were taken to establish the average error along with the standard deviation.

The following criteria were adopted for the assignment of BSA tryptic peptides. First, a particular tryptic peptide had to be detected in at least three consecutive scans. Second, if the higher abundance peptide was detected in more than 20 consecutive scans, only the isotopic distributions from 20 scans centered at the maximum of the peptide elution profile were then treated in subsequent statistical analysis. Third, tentative peptide "hits" were initially evaluated over a relatively broad mass window of $50 \mathrm{ppm}$; then observed LC elution times for the peptides were compared to the theoretically predicted values (as recently

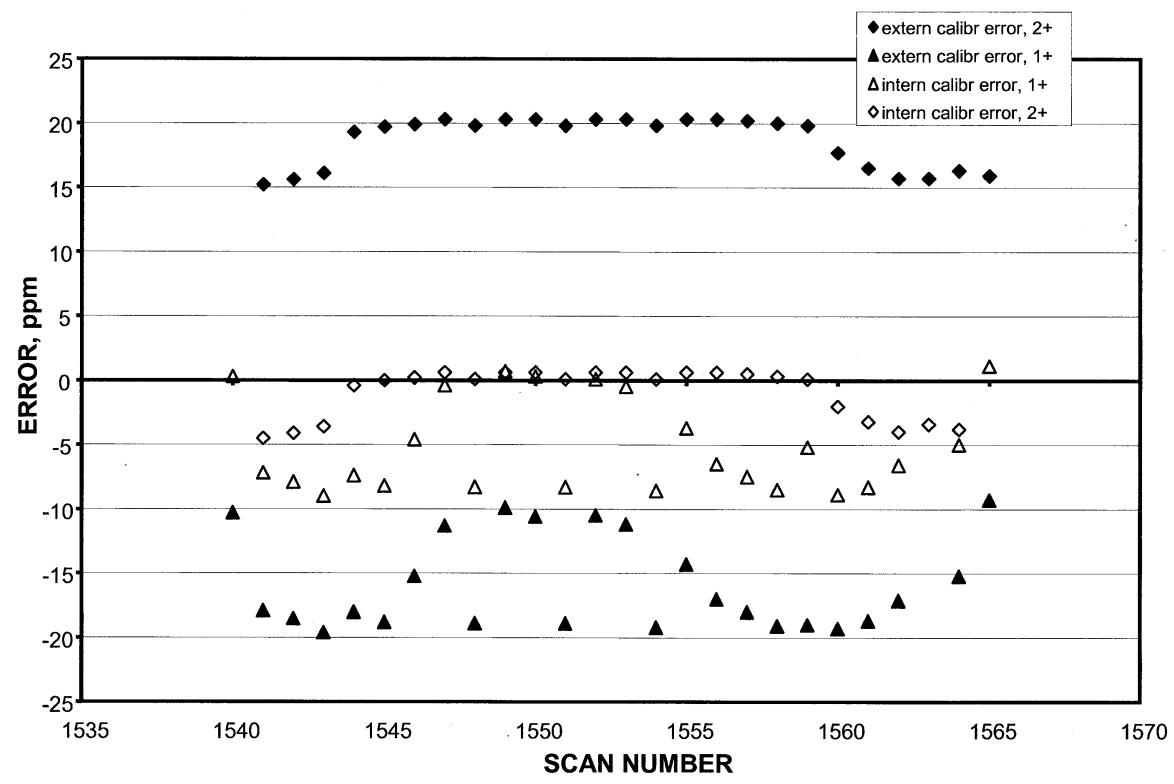

Figure 15. Dependence of the mass measurement errors on the scan number for the doubly $(2+)$ and singly $(1+)$ charged QTALVELLK peptide identified during capillary LC separation of the $0.1 \mathrm{mg} / \mathrm{mL}$ bovine serum albumin digest. Internal calibration data points were obtained using higher abundance calibrant ions. 

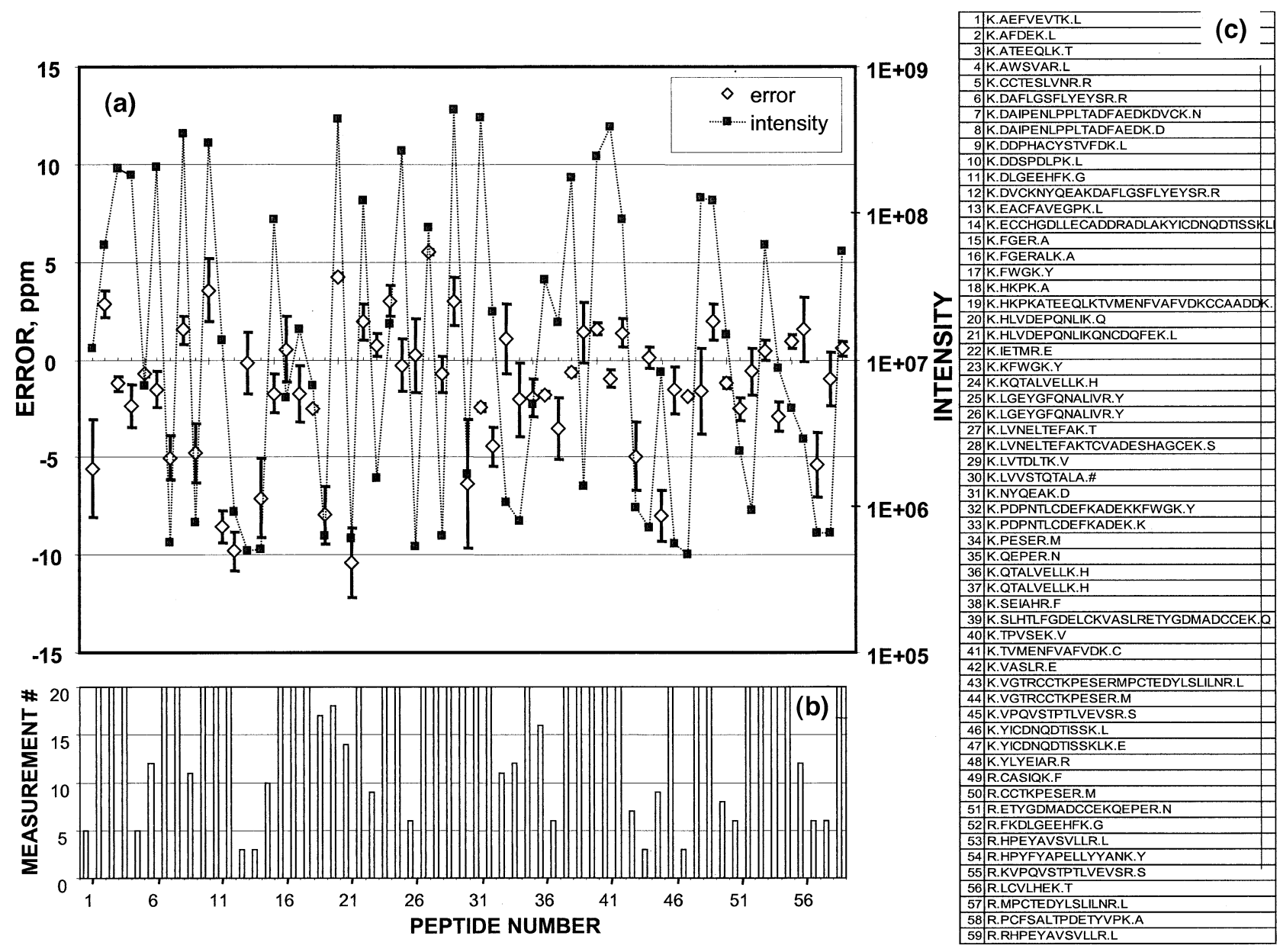

Figure 16. (a) Average errors, standard deviations and intensities for 59 peptides identified during the LC/FTICR run of the $0.1 \mathrm{mg} / \mathrm{mL}$ bovine serum albumin digest. (b) The number of measurements taken symmetrically around the maximum of the peptide elution peaks for statistical analysis of the mass measurement errors in Figure 16a as a function of the peptide number. (c) Sequences for the tryptic peptides corresponding to the peptide numbers in Figure $16 \mathbf{b}$.

developed by this laboratory [61]) and an acceptability cut-off of $+/-5 \%$. This approach proved to be effective in eliminating false positive identifications, and provided unbiased representation of the MMA for the FTICR during LC separations. The mass measurement errors for the majority of the identified peptides thus fit within a $2 \mathrm{ppm}$ window centered at $0 \mathrm{ppm}$. Interestingly, the cases of average errors larger than $5 \mathrm{ppm}$ (a few data points in Figure 16) were attributed to lower abundance peptides. Compared with the data obtained with the unmodified instrument, the number of assigned tryptic peptides increased by a factor of $\sim 3$, while the total number of isotopic distributions matching the masses of predicted BSA tryptic peptides within $10 \mathrm{ppm}$ increased by more than 5 -fold.

The increase in the number of identified peptides in the course of a capillary LC separation was primarily attributed to the following improved characteristics of the FTICR instrument. First, the higher sensitivity of the modified FTICR instrument was attained by replacing the commercial skimmer/hexapole interface with the electrodynamic ion funnel/quadrupole arrangement. Second, the development of the efficient ICR cell trapping technique resulted in more effective radial and axial confinement of the ion clouds in a shallow axial well in the middle of the ICR cell. This trapping technique provided reduced dispersion of mass measurement errors ( $<1 \mathrm{ppm}$ with external calibration) for peaks exceeding a certain intensity threshold. Third, the independent introduction of the internal calibrants through the dual electrodynamic ion funnel and the algorithm developed for internal calibration enabled reliable correction of the cyclotron frequency shifts during the LC separation and increased the MMA of the FTICR instrument by more than 5 -fold. The capabilities of the modified 9.4 tesla FTICR instrument were further improved by the implementing DREAMS and AGC technologies (to be reported elsewhere). Overall, the automated high pressure capillary LC-FTICR instrument provided the robust performance desired for high-throughput proteomics research. 


\section{Conclusions}

A capillary LC-FTICR (9.4 tesla) system was developed incorporating a front-end interface for non-selective and selective external ion accumulation comprised of a dual-ESI-emitter ion source; dual-channel electrodynamic ion funnel; collisional, selection and accumulation quadrupoles. The interface was initially evaluated in direct infusion experiments with a number of peptides/proteins as well as for capillary LC separations of proteolytic digests. A new method for the accumulation of ions in the ICR cell was also developed. The total energy of the externally (to the ICR cell) trapped ion cloud was modulated in the accumulation quadrupole and the ejected ion packets were then efficiently transferred and trapped in a shallow axial well in the middle region of the ICR cell. Under optimized conditions of ion accumulation and ejection, the trapping efficiency in the ICR cell was estimated to be close to unity. Use of the modulated-ion-energy gated trapping technique eliminated the ion-cooling step in the ICR cell without increasing magnetron radius, resulting in a significant increase in the duty cycle of the LC/ESI/FTICR instrument. To further expand the dynamic range of the instrument, selective ion ejection prior to ion accumulation was implemented. Selective ion ejection was performed using rf-only resonant dipolar excitation in the middle region of the selection quadrupole; a mass resolution of $\sim 100$ was attained, under conditions that did not sacrifice achievable sensitivity. The modified FTICR instrument was coupled to a very high pressure, high efficiency capillary LC separation and the entire LC-FTICR system was fully automated. Unattended operation of the instrument revealed the exceptional reproducibility $(1-5 \%$ deviation in elution times for peptides from a bacterial proteome, although it should be noted that these residual variations can be corrected in data analysis [61]), repeatability (10-20 \% deviation in detected abundances for peptides from the same aliquot analyzed weeks apart), and robustness (highthroughput operation for 5 months without significant downtime) of the overall LC-FTICR system. When combined with the modulated-ion-energy gated trapping, the internal calibration of FTICR mass spectra decreased the dispersion of the mass measurement errors for identification of peptides in conjunction with LC separations to high mass accuracies over a dynamic range of $10^{3}$ in each spectrum. Greater MMA can, of course, be achieved by restraining ion populations, but with somewhat reduced dynamic range.

The automated capillary LC-9.4 tesla FTICR has proven to be a robust, sensitive, accurate, high-resolution, high-dynamic-range instrument suitable for highthroughput proteomic analyses. Further developments in progress include the automation of higher performance capillary LC separations at higher pressures, and the using on smaller i.d. capillaries to further increase sensitivity and decrease or eliminate ion suppression effects [62].

\section{Acknowledgments}

The authors gratefully acknowledge Drs. Joe Meier, Steven van Orden, Daniel Meili, and John Wronka from Bruker Daltonics for their support in modifying the FTICR instrument. This research was supported by the U.S. Department of Energy, Office of Biological and Environmental Research. PNNL is a multiprogram national laboratory operated by Battelle Memorial Institute for the U.S. Department of Energy under contract DE-AC06-76RLO 1830.

\section{References}

1. Gygi, S. P.; Rochon, Y.; Franza, B. R.; Aebersold, R. Mol. Cell Biol. 1999, 19, 1720-1730.

2. O'Farrell, P. H. J. Biol. Chem. 1975, 250, 4007-4021.

3. Karas, M.; Hillenkamp, F. Anal. Chem. 1988, 60, 2299-2301.

4. Fenn, J. B.; Mann, M.; Meng, C. K.; Wong, S. K.; Whitehouse, V. Science 1989, 246, 64-71.

5. Strupat, K.; Karas, M.; Hillenkamp, F.; Eckerskorn, C.; Lottspeich, F. Anal. Chem. 1994, 66, 464-470.

6. Henzel, W. J.; Billeci, T. M.; Stults, J. T.; Wong, S. C.; Grimley, C.; Watanabe, C. Proc. Natl. Acad. Sci. U.S.A. 1993, 90, 50115015.

7. Yates, J. R., III; Speicher, S.; Griffin, P. R.; Hunkapiller, T. Anal. Biochem. 1993, 214, 397-408.

8. Mortz, E.; Vorm, O.; Mann, M.; Roepstorff, P. Biol. Mass Spetrom. 1994, 23, 249-261.

9. Shevchenko, A.; Wilm, M.; Vorm, O.; Mann, M. Anal. Chem. 1996, 68, 850-858.

10. Wilm, M.; Shevchenko, A.; Houthaeve, T.; Breit, S.; Schweigerer, L.; Fotsis, T.; Mann, M. Nature 1996, 379, 466-469.

11. Courchesne, P. L.; Luethy, R.; Patterson, S. D. Electrophoresis 1997, 18, 369-381.

12. Gygi, S. P.; Corthals, G. L.; Zhang, Y.; Rochon, Y.; Aebersold, R. Proc. Natl. Acad. Sci. U.S.A. 2000, 97, 9390-9395.

13. Sharp, P. M.; Li, W. H. Nucleic Acids Res. 1987, 15, 1281-1295.

14. McCormack, A. L.; Schieltz, D. M.; Goode, B.; Yang, S.; Barnes, G.; Drubin, D.; Yates, J. R., III. Anal. Chem. 1997, 69, 767-776.

15. Link, A. J.; Eng, J.; Schieltz, D. M.; Carmack, E.; Mize, G. J.; Morris, D. R.; Garvik, B. M.; Yates, J. R., III. Nat. Biotech. 1999, 17, 676-682.

16. Wahburn, M. P.; Wolters, D.; Yates, J. R., III. Nat. Biotech. 2001, 19, 242-247.

17. Perrot, M.; Sagliocco, F.; Mini, T.; Monribot, C.; Schneider, U.; Shevchenko, A.; Mann, M.; Jeno, P.; Boucherie, H. Electrophoresis 1999, 20, 2280-2298.

18. Yates, J. R.; Eng, J. K.; McCormack, A. L.; Schieltz, D. Anal. Chem. 1995, 67, 1426-1436.

19. Smith, R. D.; Anderson, G. A.; Lipton, M. S.; Pasa-Tolic, L.; Shen, Y.; Conrads, T. P.; Veenstra, T. D.; Udseth, H. R. Proteomics 2002, 2, 513-523.

20. Stockton, G. W.; Meek, J. T.; Millen, W. G.; Wayne, R. S. In: FT-ICR/MS: Analytical Applications of Fourier Transfrom Ion Cyclotron Resonance Mass Spectrometry; Asamoto, B., Ed.; VCH: New York, 1991; pp. 235-272.

21. Comisarow, M. B.; Marshall, A. G. Chem. Phys. Lett. 1974, 25, 282.

22. Marshall, A. G. Int. J. Mass Spectrom. 2000, 200, 331-356.

23. Belov, M. E.; Nikolaev, E. N.; Anderson, G. A.; Udseth, H. R.; Conrads, T. P.; Veenstra, T. D.; Masselon, C. D.; Gorshkov, M. V.; Smith, R. D. Anal. Chem. 2001, 73, 253-261.

24. Shen, Y.; Zhao, R.; Belov, M. E.; Conrads, T. P.; Anderson, G. A.; Tang, K.; Pasa-Tolic, L.; Veenstra, T. D.; Lipton, M. S.; Smith, R. D. Anal. Chem. 2001, 73, 1766-1775.

25. Conrads, T. P.; Alving, K.; Veenstra, T. D.; Belov, M. E.; Anderson, G. A.; Anderson, D. J.; Pasa-Tolic, L.; Chrisler, W. B.; Trall, B. D.; Smith, R. D. Anal. Chem. 2001, 73, 2132-2139. 
26. Shen, Y.; Tolic, N.; Zao, R.; Pasa-Tolic, L.; Li, L.; Berger, S. J.; Harkewicz, R.; Anderson, G. A.; Belov, M. E.; Smith, R. D. Anal. Chem. 2001, 73, 3011-3021.

27. Senko, M. W.; Hendrikson, C. L.; Emmett, M. R.; Shi, S. D.-H.; Marshall, A. G. J. Am. Soc. Mass Spectrom. 1997, 8, 970-976.

28. McIver, R. US Patent 4,535,235; 1985.

29. Beu, S. C.; Laude, D. A., Jr. Int. J. Mass Spetrom. Ion Processes 1991, 104, 109-127.

30. Belov, M. E.; Nikolaev, E. N.; Anderson, G. A.; Auberry, K. J.; Harkewicz, R.; Smith, R. D. J. Am. Soc. Mass Spectrom. 2001, 12, $38-48$.

31. Paul, W.; Reinhard, H. P.; von Zahn, U. Z. Physik 1958, 152, 143-147.

32. Campbell, J. M.; Collings, B. A.; Douglas, D. J. Rapid Commun. Mass Spectrom. 1998, 12, 1463-1474.

33. Quenzer, T. L.; Emmet, M. R.; Hendrickson, C. L.; Kelly, P. H.; Marshall, A. G. Anal. Chem. 2001, 73, 1721-1725.

34. Belov, M. E.; Anderson, G. A.; Angell, N. H.; Shen, Y.; Tolic, N.; Smith, R. D. Anal. Chem. 2001, 73, 1552-1560.

35. Pasa-Tolic, L.; Harkewicz, R.; Anderson, G. A.; Tolic, N.; Shen, Y. F.; Zhao, R.; Thrall, B.; Masselon, C.; Smith, R. D. J. Am. Soc. Mass Spectrom. 2002, 13, 954-963.

36. Shi, S. D.-H.; Hendrickson, C. L.; Marshall, A. G. Proc. Natl. Acad. Sci. U.S.A. 1998, 95, 11532-11537.

37. Gorshkov, M. V.; Guan, S.; Marshall, A. G. Int. J. Mass Spectrom. Ion Processes 1993, 128, 47-60.

38. Gorshkov, M. V.; Masselon, C. D.; Anderson, G. A.; Udseth, H. R.; Harkewicz, R.; Smith, R. D. J. Am. Soc. Mass Spectrom. 2001, 12, 1169-1173.

39. Qian, K.; Rodgers, R. P.; Hendrickson, C. L.; Emmett, M. R.; Marshall, A. G. Proceedings of the 48th ASMS Conference on Mass Spectrometry and Allied Topics; Long Beach, CA, 2000.

40. Futcher, B.; Latter, G. I.; Monardo, P.; McLaughlin, C. S.; Garrel, J. I. Mol. Cell Biol. 1999, 19, 7357-7368.

41. Corthals, G. L.; Wasinger, V. C.; Hochstrasser, D. F.; Sanchez, J. C. Electrophoresis 2000, 21, 1105-1115.

42. Belov, M. E.; Zhang, R.; Strittmatter, E. F.; Prior, D. C.; Tang, K.; Smith, R. D. Anal. Chem. 2003, 75, 4195-4205.

43. Conrads, T. P.; Anderson, G. A.; Veenstra, T. D.; Pasa-Tolic, L.; Smith, R. D. Anal. Chem. 2000, 72, 3349-3354.

44. Lipton, M. S.; Pasa-Tolic, L.; Anderson, G. A.; Anderson, D. J.; Auberry, D. L.; Battista, J. R.; Daly, M. J.; Fredrickson, J.;
Hixson, K. K.; Kostandarithes, H.; Masselon, C.; Markillie, L. M.; Moore, R.; Romine, M. F.; Shen, Y.; Strittmatter, E.; Tolic, N.; Udseth, H. R.; Venkateswaran, A.; Wong, K. K.; Zhao, R.; Smith, R. D. Proc. Natl. Acad. Sci. U.S.A. 2002, 99, 11049-11054.

45. Belov, M. E.; Gorshkov, M. V.; Udseth, H. R.; Anderson, G. A.; Tolmachev, A. V.; Prior, D. C.; Harkewicz, R.; Smith, R. D. J. Am Soc. Mass Spectrom. 2000, 11, 19-23.

46. Tang, K.; Tolmachev, A. V.; Zhang, R.; Nikolaev, E. N.; Belov, M. E.; Udseth, H. R.; Smith, R. D. Anal.Chem. 2002, 74, 5431-5437.

47. Beijerinck, H. C. W.; Verster, N. F. Physica B and C 1981, 111, 327-352.

48. Cha, B.; Blades, M.; Douglas, D. J. Anal. Chem. 2000, 72, 5647-5654.

49. Belov, M. E.; Anderson, G. A.; Smith, R. D. Int. J. Mass Spectrom. 2002, 218, 269-275.

50. Caravatti, P.; Allemann, M. Org. Mass Spectrom. 1991, 26 , 514-518.

51. Henry, K. D.; Williams, E. R.; Wang, B. H.; McLafferty, F. W.; Shabanowitz, J.; Hunt, D. F. Proc. Natl. Acad. Sci. U.S.A. 1989, 86, 9075.

52. Flanagan, J. M. US Patent 5,872,357; 1999.

53. Kofel, P.; Allemann, M.; Kellerhals, H.; Wanczek, K.-P. Int. J. Mass Spectrom. Ion Processes 1990, 98, 1-24.

54. Caravatti, P. U.S. Patent 4,924,089; 1990.

55. Hogan, J. D.; Laude, D. A., Jr. Anal. Chem. 1990, 62, 530-535.

56. Beu, S. C.; Laude, D. A., Jr. Int. J. Mass Spectrom. 1991, 108, 255-268.

57. Li, G.-Z.; Guan, S.; Marshall, A. G. J. Am. Soc. Mass Spectrom. $1997,8,793-800$.

58. Hannis, J. C.; Muddiman, D. C. J. Am. Soc. Mass Spectrom. 2000, 11, 876-883.

59. Ledford, E. B.; Rempel, D. L.; Gross, M. L. Anal. Chem. 1984, 56, 2744-2748

60. Boggs, P. T.; Donaldson, J. R.; Byrd, R. H.; Schnabel, R. B. ACM T. Math Software 1998, 15, 348-364.

61. Petritis, K.; Kangas, L. J.; Ferguson, P. L.; Anderson, G. A.; Pasa-Tolic, L.; Lipton, M. S.; Auberry, K. J.; Strittmatter, E.; Shen, Y.; Zhao, R.; Smith, R. D. Anal. Chem. 2003, 75, 10391048.

62. Shen, Y.; Zhao, R.; Berger, S. J.; Anderson, G. A.; Rodriguez, N.; Smith, R. D. Anal. Chem. 2002, 74, 4235-4249. 